Publ. RIMS, Kyoto Univ.

23 (1987), 737-769

\title{
Outer Conjugacy for Actions of Continuous Amenable Groups
}

By

\author{
Valentin Ya. GolodeTS* and Sergey D. SinelSHChIKOV**
}

\begin{abstract}
The paper introduces a cohomological approach to the outer conjugacy problem in ergodic theory. Specifically, the following fact is proved: up to an isomorphism of an approximately finite type II ergodic full group there exists only one cohomological class of cocycles with dense range in a given amenable group. This result is used to establish outer conjugacy for strictly outer actions of continuous unimodular amenable subgroups of the normalizer of the full group generated by an ergodic type II automorphism. As a special case we show that outer conjugacy of compact groups reduces merely to conjugation. Besides that the correlation is established between the automorphisms of a principal groupoid with continuous orbits and ones of its discrete reduction. Also the correspondence is found between the automorphisms of groupoids with continuous orbits and those of the associated von Neumann algebras.
\end{abstract}

\section{$\S 0$. Introduction}

The classification of Lebesgue space automorphisms is one of the central problems in ergodic theory. Initially the notion of conjugacy (or just isomorphism) of the measure space transformations was imposed. Among other well known invariants of conjugacy one should mention first of all the entropy introduced by Kolmogorov and Sinai [7]. This permits the complete classification of Bernoulli shifts to be obtained [27]. However, a large collection of non-isomorphic transformations hints that a

Communicated by H. Araki, May 26, 1986. Revised February 25, 1987.

* Institute for Low Temperature Physics and Engineering, UkrSSR Academy of Sciences, Lenin avenue, 47, Kharkov 310164, USSR.

** Kharkov State University, Department of Mechanics and Mathematics, Dzerzhinsky square 4, Kharkov 310077, USSR. 
simple complete system of invariants for conjugacy can hardly be found.

It became clear later that it is important to study the orbit equivalence for actions of (possibly different) groups on a Lebesgue space. A significant progress in this sphere was advanced in works of W. Krieger [22] and T. Hamachi, Yu. Oka, M. Osikawa [17]. Now one has that the ergodic actions of any two discrete amenable groups with a finite invariant measure are orbit equivalent [5]. A similar result is valid also for a class of continuous amenable unimodular locally compact separable (1.c.s.) groups [28].

As soon as the orbit equivalence classes of amenable group actions were described, the problem of studying the outer automorphism groups of amenable equivalence relations came up, and first of all the problem of outer conjugacy of such groups (see the definition in $\S 3$ ). The first result in this field was obtained by A. Connes and W. Krieger [6], where they described the complete system of invariants for outer conjugacy of $Z$-actions in the normalizer of the full approximately finite group $[\Gamma]$ in the case when the $\Gamma$-action is of type II. After that a similar problem was solved for a transformation group $\Gamma$ of type III [1], and in [2], [3] a complete system of invariants was obtained for actions of countable amenable groups in the normalizer of an approximately finite ergodic full group of an arbitrary type. The outer conjugacy for actions of countable groups were also studied in [33]. The outer conjugacy problem for continuous transformation groups was never considered before.

It should be noted that the outer conjugacy is also extensively studied for automorphism groups of von Neumann algebras (see for instance [4], [21], [26], [35]). However, for the case of continuous groups, except compact Abelian ones [21], the worthwhile results are still unknown.

We approach the studying of outer conjugacy problem by proving the uniqueness theorem for cocycles of an ergodic type II automorphism with dense ranges (see [14] and Theorem 1.5 of this paper). This theorem is also of some independent interest. It claims that up to an automorphism of the full group there exists only one cohomological class of cocycles with dense ranges in a fixed amenable group.

In $\S 2$ we establish a correspondence between the automorphisms of measure groupoids with continuous orbits and ones of their discrete reductions and the associated von Neumann algebras (see Theorems 2.4, 2.7 -2.8 ). We also describe how to form the semidirect product of a measure 
groupoid with discrete orbits by a continuous 1.c.s. group of its non-strict automorphisms (Remark 2.2).

$\S 3$ contains the proof of the main result concerning outer conjugacy for strictly outer actions of continuous unimodular amenable l.c.s. groups (Theorem 3.3). The case of compact groups is considered separately in $\S 4$. It will be shown that outer conjugacy of such groups reduces merely to conjugation by means of some transformation from the full group normalizer (Theorem 4.1; cf. [38]).

Finally, we prove in Appendix that the equivalence relation generated by an ergodic automorphism $T$ together with an amenable subgroup of $N[T]$, is approximately finite (Theorem A1).

The present paper is a mildly expanded version of our preprint [37].

\section{$\S 1$. Uniqueness Theorem for Cocycles with $\mathbb{D e n s e} \mathbb{R}$ anges}

We begin with recalling some definitions. Let $(S, \mu)$ be a Lebesgue space and $\mathscr{D}$ a non-singular countable transformation group of $(S, \mu)$. Then one can consider the full group [D] of automorphisms $\gamma$ on $(S, \mu)$ such that $\gamma_{s} \in\{\omega s: \omega \in \mathscr{D}\}$ for a.a. $s \in S$ [6]. In the case when $\mathscr{D}$ is generated by a single transformation $T$ we shall write $[T]$ to denote the corresponding full group. The $\mathscr{D}$-action on $S$ and the full group [D] are said to be of type $\mathrm{II}_{1}\left(\mathrm{II}_{\infty}\right)$ if there exists a finite (infinite ) $\mathscr{D}$-invariant measure, equivalent to $\mu$.

The normalizer $N[\mathscr{D}]$ of the full group [D] is formed by the automorphisms $\theta \in$ Aut $(S, \mu)$ with the property $\theta^{-1}[\mathscr{D}] \theta=[\mathscr{D}]$. Assume that $[\mathscr{D}]$ is of type $\mathrm{II}_{1}$, and $\mu$ is $\mathscr{D}$-invariant, then any $\theta \in N[\mathscr{D}]$ is $\mu$-preserving. In the case of type $\mathrm{II}_{\infty}$ one has $\mu^{\circ} \theta=(\bmod \theta) \cdot \mu$ for some positive number $\bmod \theta[6]$.

Suppose a l.c.s. group $H$ acts ergodically on a Lebesgue space $(X, \nu)$. A Borel map $\pi: H \times X \rightarrow G$ is called a cocycle of a dynamical system $(X, \nu$, $H)$ with values in a l.c.s. group $G$ if $\pi\left(h_{2} h_{1}, x\right)=\pi\left(h_{2}, h_{1} x\right) \pi\left(h_{1}, x\right)$ for all $h_{1}$, $h_{2} \in H$ at a.a. $x \in X$. Two cocycles $\pi$ and $\tau$ are said to be cohomologous if $\tau(h, x)=g(h x)^{-1} \pi(h, x) g(x)$ for some Borel function $g: X \rightarrow G$ for all $h \in$ $H$ at a.a. $x \in X$.

Let $\mu_{G}$ be the left Haar measure for $G$, then one can define a $H$-action on $\left(G \times X, \mu_{G} \times \nu\right)$ as follows: $h(g, x)=(\pi(h, x) g, h x)$ for $h \in H,(g, x) \in G$ $\times X$. We call it the skew produc 0 action and denote by $G \times{ }_{\pi} X$. In the 
case when this action is ergodic, the cocycle $\pi$ is said to have a dense range in $G$ [24].

Let $Y=\{0,1\}$ be a two element set with a measure $\nu$ defined by $\nu(\{0\})$ $=\nu(\{1\})=1 / 2$, then one can form a Lebesgue space $(X, \mu)=(Y, \nu)^{N}$ with $N$ being the set of positive integers. $(X, \mu)$ admits a countable Abelian measure preserving transformation group $\Gamma$ generated by the automorphisms $\delta_{k}, k \in N$, given by

$$
\left(\delta_{k} x\right)_{i}= \begin{cases}x_{i} & i \neq k \\ x_{i}+1(\bmod 2) & i=k\end{cases}
$$

for every sequence of 0 's and 1's $x \in X$.

The dynamical system $(X, \mu, \Gamma)$ is orbit equivalent to an ergodic type $\mathrm{II}_{1}$ automorphism [34], [5], and hence every cocycle of the latter can be transferred to $(X, \mu, \Gamma)$. Let $G$ be a l.c.s. group, then by [13, Theorem 2 of $\S 1$ ] every cocycle $c: \Gamma \times X \rightarrow G$ is completely determined by a sequence of Borel maps $f_{k}: X \rightarrow G, k \in N$ :

$$
\begin{aligned}
c\left(\delta_{k}, x\right)= & f_{1}\left(\delta_{k} x\right)^{x_{1}} \cdot f_{2}\left(\delta_{k} x\right)^{x_{2}} \cdots f_{k-1}\left(\delta_{k} x\right)^{x_{k-1}} \\
& \times f_{k}(x)^{(-1)^{x_{k}}} f_{k-1}(x)^{-x_{k-1} \cdots} f_{1}(x)^{-x_{1}}
\end{aligned}
$$

with every $f_{k}(x)$ being invariant with respect to $\delta_{1}, \cdots, \delta_{k}$. Conversely, every sequence of Borel maps $f_{k}: X \rightarrow G$ satisfying the invariance condition as above generates some cocycle $c$ in correspondence with (1.1).

Definition 1.1. A cocycle $c: \Gamma \times X \rightarrow G$, determined by a sequence of Borel functions $f_{k}: X \rightarrow G$ (see (1.1)), is said to have a special form if the following conditions are satisfied:

(i) every function $f_{k}(x)$ takes only finitely many values;

(ii) the inverse images for $f_{k}$ are the finite unions of cylinder sets;

(iii) there exists a sequence $\left\{m_{i}\right\}_{i=1}^{\infty} \subset \mathbb{N}$ such that $f_{k}\left(\delta_{j} x\right)=f_{k}(x)$ for $k \leq$ $m_{i}<j$ or, equivalently, $f_{k}(x)$ depends only on $x_{k+1}, \cdots, x_{m_{2}}$ with $i$ being chosen so that $m_{\imath-1}<k \leq m_{i}$.

Since the $\Gamma$-action on $X$ is free, every cocycle of this action can be uniquely extended up to a cocycle of the full group $[\Gamma]$. Conversely, given any countable freely acting subgroup $\Gamma^{\prime} \subset[\Gamma]$ with $\left[\Gamma^{\prime}\right]=[\Gamma]$, then every cocycle of $[\Gamma]$ is an extension as above of some cocycle of the $\Gamma^{\prime}$-action on $X$. We shall show that every cohomology class of cocycles with dense range contains a cocycle of the special form relatively to some freely acting 
$\Gamma^{\prime} \subset[\Gamma]$ such that $\Gamma^{\prime}$ is isomorphic to $\Gamma$ and $\left[\Gamma^{\prime}\right]=[\Gamma]$.

Below in this section we shall use for a l.c.s. group $G$ a notion $\left\{W_{i}\right\}_{i=1}^{\infty}$ which refers to the fundamental system of neighbourhoods of the identity in $G$ with the following properties: every $W_{i}$ is compact, $W_{i}=W_{i}^{-1}$, and $W_{i+1} \cdot W_{i+1} \subset W_{i}$.

Let $\alpha$ and $\beta$ be the cocycles of $(X, \mu, \Gamma)$ determined by sequences of functions $f_{k}: X \rightarrow G$ and $f_{k}: X \rightarrow G$ respectively. Set up

$$
\begin{aligned}
& \varphi_{n}(x)=f_{n}(x)^{-x_{n}} f_{n-1}(x)^{-x_{n-1}} \cdots f_{1}(x)^{-x_{1}}, \\
& \bar{\varphi}_{n}(x)=\bar{f}_{n}(x)^{-x_{n}} \bar{f}_{n}(x)^{-x_{n-1}} \ldots \bar{f}_{1}(x)^{-x_{1}} .
\end{aligned}
$$

Lemma 1.2. Suppose that one can associate to every pair of functions $f_{n}(x)$ and $\bar{f}_{n}(x)$ a set $A_{n} \subset X$ and a neighbourhood of the identity $V_{n} \subset G$ such that $\mu\left(A_{n}\right)>1-1 / 2^{n}$ and for $x \in A_{n}$

$$
\begin{aligned}
& \varphi_{n-1}(x)^{-1} V_{n} \varphi_{n-1}(x) \subset W_{n}, \\
& f_{n}(x) \cdot \bar{f}_{n}(x)^{-1} \in V_{n} .
\end{aligned}
$$

Then $\alpha$ and $\beta$ are cohomologous.

Proof. Let $B_{n}=\bigcap_{i=n}^{\infty} A_{i}$, then $\mu\left(B_{n}\right)>1-1 / 2^{n}$. Form a sequence of functions $g_{n}(x)=\varphi_{n}(x)^{-1} \bar{\varphi}_{n}(x)$. Now for all $n, k \in N, x \in B_{n}$ we have:

$$
\begin{aligned}
& g_{n+k}(x) g_{n}(x)^{-1}=\varphi_{n+k}(x)^{-1} \bar{\varphi}_{n+k}(x) \bar{\varphi}_{n}(x)^{-1} \varphi_{n}(x) \\
& =\varphi_{n}(x)^{-1} f_{n+1}(x)^{x_{n+1}} \cdots f_{n+k}(x)^{x_{n+k}} \bar{f}_{n+k}(x)^{-x_{n+k} \ldots} \bar{f}_{n+1}(x)^{-x_{n+1}} \varphi_{n}(x) \\
& \in W_{n+k} \varphi_{n}(x)^{-1} f_{n+1}(x)^{x_{n+1} \cdots} f_{n+k-1}(x)^{x_{n+k-1}} \bar{f}_{n+k-1}(x)^{-x_{n+k-1}} \\
& \cdots \bar{f}_{n+1}(x)^{-x_{n+1}} \varphi_{n}(x) \\
& \subset \cdots \subset W_{n+k} W_{n+k-1} \cdots W_{n+1} \subset W_{n+k-1} W_{n+k-1} W_{n+k-2} \cdots W_{n+1} \subset \\
& \subset W_{n+k-2} W_{n+k-2} W_{n+k-3} \cdots W_{n+1} \subset \cdots \subset W_{n} .
\end{aligned}
$$

Since $B_{n} \subset B_{n+1}$ and $\bigcup_{n=1}^{\infty} B_{n}=X(\bmod 0)$, the correlation $g_{n+k}(x) g_{n}(x)^{-1}$ $\in W_{n}$ for $x \in B_{n}$ implies that $g_{n}(x)$ converge a.e. to a function $g(x)$ as $n \rightarrow \infty$. Let $n \geq k$, then

$$
\begin{aligned}
g_{n}\left(\delta_{k} x\right) \cdot \beta\left(\delta_{k}, x\right) \cdot g_{n}(x)^{-1} \\
=\varphi_{n}\left(\delta_{k} x\right)^{-1} \bar{\varphi}_{n}\left(\delta_{k} x\right) \bar{\varphi}_{k-1}\left(\delta_{k} x\right)^{-1} \cdot \bar{f}_{k}(x)^{(-1) x_{k}} \bar{\varphi}_{k-1}(x) \bar{\varphi}_{n}(x)^{-1} \varphi_{n}(x) \\
=\varphi_{n}\left(\delta_{k} x\right)^{-1} \cdot \bar{f}_{n}\left(\delta_{k} x\right)^{-x_{n} \ldots} \bar{f}_{k}\left(\delta_{k} x\right)^{-\left(x_{k}+1\right)} \bar{f}_{k}(x)^{(-1) x_{k}} \\
\quad \times \bar{f}_{k}(x)^{x_{k} \ldots} \bar{f}_{n}(x)^{x_{n}} \varphi_{n}(x)
\end{aligned}
$$




$$
\begin{aligned}
& =\varphi_{n}\left(\delta_{k} x\right)^{-1} \cdot \varphi_{n}(x)=\varphi_{k}\left(\delta_{k} x\right)^{-1} \cdot \varphi_{k}(x) \\
& =\varphi_{k-1}\left(\delta_{k} x\right)^{-1} \cdot f_{k}(x)^{(-1) x_{k}} \varphi_{k-1}(x)=\alpha\left(\delta_{k}, x\right) .
\end{aligned}
$$

Passing in the correlation

$$
\alpha\left(\delta_{k}, x\right)=g_{n}\left(\delta_{k} x\right) \cdot \beta\left(\delta_{k}, x\right) \cdot g_{n}(x)^{-1}
$$

we have got to a limit as $n \rightarrow \infty$, we obtain for a.a. $x \in X$ and all $k \in \mathbb{N}$

$$
\alpha\left(\delta_{k}, x\right)=g\left(\delta_{k} x\right) \cdot \beta\left(\delta_{k}, x\right) \cdot g(x)^{-1},
$$

i.e. $\alpha$ is cohomologous to $\beta$.

Q.E.D.

Lemma 1.3. Every cocycle $\alpha: \Gamma \times X \rightarrow G$ can be replaced by a cohomologous cocycle $\beta$ with values in a given countable dense subgroup $H \subset$ G.

Proof. Suppose $\alpha$ is determined by the functions $f_{k}: X \rightarrow G$. Since $G$ is a countable union of compact sets, one can associate to each function $f_{n}(x)$ a set $A_{n} \subset X$ which is invariant with respect to $\delta_{1}, \cdots, \delta_{n}, \mu\left(A_{n}\right)>1$ $-1 / 2^{n}$, and a compact set $Q_{n} \subset G$ containing the identity so that $f_{n}(x) \in Q_{n}$ for $x \in A_{n}$. Then choose a neighbourhood of the identity $V_{n}$ so that for each element $h$ of a compact set $Q_{1} \cdot Q_{2} \cdots Q_{n-1}$ one has $h V_{n} h^{-1} \subset W_{n}(n \geq 2$; $\left.V_{1}=W_{1}\right)$. Approximate $f_{n}(x)$ by a function $\bar{f}_{n}(x)$ with values in $H$ so that $f_{n}(x) \bar{f}_{n}(x)^{-1} \in V_{n}$, and $\bar{f}_{n}\left(\delta_{j} x\right)=\bar{f}_{n}(x)$ for $1 \leq j \leq n$. Thereby we are in the conditions of Lemma 1.2 , so that if $\beta$ is a cocycle determined by the functions $\bar{f}_{n}(x)$, then $\beta$ is cohomologous to $\alpha$.

Q.E.D.

Consider a metric $d$ on a full group $[\Gamma]$ :

$$
d\left(\omega_{1}, \omega_{2}\right)=\mu\left(\left\{x: \omega_{1} x \neq \omega_{2} x\right\}\right) \text { for } \omega_{1}, \omega_{2} \in[\Gamma] .
$$

Then $([\Gamma], d)$ is a complete metric space [8].

Lemma 1.4. Let $\alpha$ be a cocycle of the dynamical system $(X, \mu, \Gamma)$ with dense range in a l.c.s. group $G$. Then the cohomology class of a contains a cocycle $\pi$ admitting a representation in a special form.

Proof. One may assume by Lemma 1.3 that $\alpha$ takes values in a countable dense subgroup $H \subset G$.

Let $\left\{B_{i}\right\}_{i=1}^{\infty}$ be a sequence of Borel subsets in $X$ generating the Borel $\sigma$-algebra, and suppose that every set appears in this sequence infinitely often. Form in a similar way a sequence $\left\{\gamma_{i}\right\}_{i=1}^{\infty}$ consisting of generators of the group $\Gamma$ so that each generator appears in the sequence infinitely often. 
We shall construct pairwise commuting automorphisms $\hat{\delta}_{i} \in[\Gamma](i \in \mathbb{N})$ with order 2, which generate $[\Gamma]$, and associate to each of them a function $p_{1}: X \rightarrow H$. These will determine a cocycle $\pi$ in correspondence with (1.1).

By [8, Lemma 6.3] we can choose a cyclic subgroup $L_{1}$ with order $2^{a}$ in $[\Gamma]$ so that $d\left(\left[L_{1}\right], \gamma_{1}\right)<1 / 2$. Then there exist pairwise commuting automorphisms $\hat{\delta}_{1}, \cdots, \hat{\delta}_{a} \in[\Gamma]$, each of them with order 2 , which generate the full group coincident with $\left[L_{1}\right][8$, Lemma 6.4$]$. We can associate to everyone of these automorphisms a function $\widehat{f}_{i}: X \rightarrow H$ so that

$$
\begin{array}{lll}
\widehat{f}_{i}(x)=\alpha\left(\widehat{\delta}_{i}, x\right) & \text { for } & x \in F_{i}, \text { and } \\
\widehat{f}_{i}\left(\widehat{\delta}_{j} x\right)=\widehat{f}_{i}(x) & \text { for } & 1 \leq j \leq i,
\end{array}
$$

with $F_{2}$ being a fundamental set of the group $\hat{\Gamma}_{2}$ generated by $\hat{\delta}_{1}, \cdots, \hat{\delta}_{2}$, $\mu\left(F_{2}\right)=1 / 2^{i}$.

Choose for every function $\widehat{f}_{2}(x), 1 \leq i \leq a$, a finite set $Q_{2} \subset H$ containing the identity and a set $A_{2} \subset X$ invariant with respect to $\bar{\delta}_{1}, \cdots, \widehat{\delta}_{2}$ so that

$$
\begin{aligned}
& \mu\left(A_{i}\right)>1-1 / 2^{i+1} \\
& \widehat{f}_{i}(x) \in Q_{i} \text { for } \quad x \in A_{i} .
\end{aligned}
$$

For $i$ as above set

$$
p_{i}^{\prime}(x)=\left\{\begin{array}{lll}
\hat{f}_{i}(x) & \text { for } & x \in A_{i} \\
e & \text { for } & x \in X \backslash A_{i} .
\end{array}\right.
$$

Everyone of these functions takes only finitely many values.

Let $X=\bigcup_{k=1}^{s} \mathscr{D}_{k}$ be a partition of $X$ generated by the inverse images of $p_{i}{ }^{\prime}(x), i=1, \cdots, a$. Then the sets

$$
\gamma^{-1}\left(\mathscr{D}_{k} \cap \gamma F_{a}\right) \text { and } \gamma^{-1}\left(B_{1} \cap \gamma F_{a}\right), \quad k=1, \cdots, s, \gamma \in \hat{\Gamma}_{a},
$$

generate also a partition of $F_{a}$, say $F_{a}=\bigcup_{j=1}^{N} R_{j}$. Let $p$ be a positive integer such that $2^{p}>2^{a+1} N$. Represent $R_{j}, 1 \leq j \leq N$, as a disjoint union $R_{j}=\bigcup_{t=1}^{N_{j}} R_{j}{ }^{t}$ so that $\mu\left(R_{j}{ }^{t}\right)=1 / 2^{p}$ for $1 \leq t \leq N_{j}-1$, and $\mu\left(R_{j}^{N_{j}}\right)<1 / 2^{p}$. Denote $\bar{\Phi}=$ $\bigcup_{\gamma \in \Gamma a j=1}^{N} \gamma R_{j}^{N,}$ and set for $1 \leq i \leq a$

$$
p_{i}(x)= \begin{cases}p_{i}{ }^{\prime}(x), & x \in X \backslash \Phi \\ e & , \quad x \in \Phi\end{cases}
$$


It follows from (1.6) and (1.7) that $p_{i}(x) \neq \hat{f}_{i}(x)$ on a set with measure less than $1 / 2^{i+1}+1 / 2^{a+1} \leq 1 / 2^{i}$.

One can readily transform $\bigcup_{j=1}^{N} R_{j}^{N_{j}}$ into the finite union of disjoint sets with measure $1 / 2^{p}$ which form together with $R_{j}{ }^{t}, 1 \leq j \leq N, 1 \leq t \leq N_{j}-1$, a partition of $F_{a}$ by $2^{p-a}$ disjoint sets $M_{j}, 0 \leq j \leq 2^{p-a}-1$.

Let $U$ be a symmetric neighbourhood of the identity in $G$ such that for all elements $h$ of the finite set $Q_{1} \cdot Q_{2} \cdots Q_{a}$ (see (1.4), (1.5)) one has $h U h^{-1} \subset$ $W_{p}$. Choose a symmetric neighbourhood of the identity $V$ so that

$$
\underbrace{V \cdot V \cdot \cdots V}_{2^{p-a} \text { times }} \subset U \text {. }
$$

Since $\alpha$ has dense range in $G$, a simple argument permits one to construct a periodic automorphism $\zeta$ on $F_{a}$ from the full group $[\Gamma]$ reduced to $F_{a}$, with order $2^{p-a}$, which permutes $M_{j}, 0 \leq j \leq 2^{p-a}-1$, and such that $\alpha(\zeta$, $x) \in V$ for a.a. $x \in F_{a}$. We shall describe this construction in more details.

Let $V^{\prime}$ be a symmetric neighbourhood of the identity in $G$ satisfying the condition $V^{\prime} \cdot V^{\prime} \subset V$. It follows from the ergodicity of the skew product action $G \times{ }_{a} X$ that there exist a set $E_{1} \subset M_{0}$ and an element $\gamma_{1} \in \Gamma$ such that $\mu\left(E_{1}\right)>0, \gamma_{1} E_{1} \subset M_{1}$, and $\alpha\left(\gamma_{1}, x\right) \in V^{\prime}$ for $x \in E_{1}$. Then we form in a similar way $E_{2} \subset M_{0} \backslash E_{1}$ and $\gamma_{2} \in \Gamma$ so that $\mu\left(E_{2}\right)>0, \gamma_{2} E_{2} \subset M_{1} \backslash \gamma_{1} E_{1}$, and $\alpha\left(\gamma_{2}, x\right) \in V^{\prime}$ for $x \in E_{2}$. Repeat this procedure infinitely many times and thus form a Borel isomorphism $\omega_{1}: M_{0} \rightarrow M_{1}(\bmod 0)$ from the full group $[\Gamma]$ such that $\omega_{1} x=\gamma_{i} x$ for $x \in E_{i}$, and $\alpha\left(\omega_{1}, x\right) \in V^{\prime}$ at a.a. $x \in M_{0}$.

After that we construct in a similar manner isomorphisms $\omega_{k}: M_{0} \rightarrow M_{k}$, $2 \leq K \leq 2^{p-a}-1$; let also $\omega_{0}=\operatorname{id}_{M_{0}}$. Define an isomorphism $\zeta: \zeta x=\omega_{k+1} \omega_{k}{ }^{-1} x$ for $x \in M_{k}$ with indices being taken mod $2^{p-a}$. It is straightforward to see that $\zeta$ satisfies all necessary conditions; in particular,

$$
\begin{aligned}
\alpha(\zeta, x) & =\alpha\left(\omega_{k+1} \omega_{k}^{-1}, x\right)=\alpha\left(\omega_{k+1}, \omega_{k}{ }^{-1} x\right) \cdot \alpha\left(\omega_{k}{ }^{-1}, x\right) \\
& =\alpha\left(\omega_{k+1}, \omega_{k}{ }^{-1} x\right) \cdot \alpha\left(\omega_{k}, \omega_{k}{ }^{-1} x\right)^{-1} \in V^{\prime} \cdot V^{\prime} \subset V
\end{aligned}
$$

under an appropriate choice of $k$.

Construct as before related to $\zeta$ automorphisms $\bar{\delta}_{i}$ on $F_{a}$, with order 2, $a+1 \leq i \leq p$, and then extend them onto $\gamma F_{a}$ for every $\gamma \in \hat{\Gamma}_{a}$ by formulae $\gamma \widehat{\delta}_{i} \gamma^{-1}$. Thus we obtain new automorphisms with order 2 on $X$ which commute in pairs with each other and with formed before $\bar{\delta}_{i}$ 's. As above, associate with newly formed transformations the functions $\hat{f}_{i}: X \rightarrow G$ (see 
(1.2), (1.3)). Then $\hat{f}_{i}(x) \in U$ at a.a. $x \in X$, as one can deduce from the properties of $\zeta$ and (1.8). Set $p_{i}(x)=e$ for $x \in X, a+1 \leq i \leq p$, then $\widehat{f}_{i}(x)^{-1}$ - $p_{i}(x) \in U$ at a.a. $x \in X$.

It follows from our construction that by the choice of $p$ the set $B_{1}$ is approximated by the $\hat{\Gamma}_{p}$-invariant $\sigma$-algebra generated by $F_{p}$ in measure up to $1 / 2$. This completes the first step of our construction $\left(m_{2}=p\right)$.

Repeat this procedure making the approximation more and more exact.

At the $n$-th step $\gamma_{n}$ is approximated in the metric $d$ by the group [ $\left.L_{n}\right]$ up to $1 / 2^{n}$. This provides $\left[\bigcup_{i=1}^{\infty} \hat{\Gamma}_{i}\right]=[\Gamma]$. Furthermore, at the $n$-th step $B_{n}$ is approximated in measure by the $\widehat{\Gamma}_{m_{n}}$-invariant finite $\sigma$-algebra generated by $F_{m_{n}}$ up to $1 / 2^{n}$. This guarantees the coincidence of the initial Borel $\sigma$-algebra on $X$ with the $\sigma$-algebra generated by the increasing sequence of finite $\sigma$-algebras associated with $\widehat{\Gamma}_{m_{n}}, n \in \mathbb{N}$.

The functions $\hat{f}_{2}(x)$ and $p_{i}(x)$ formed above satisfy the conditions of Lemma 1.2 , so that if we denote by $\pi$ the cocycle determined by $\left\{p_{i}(x)\right\}_{2=1}^{\infty}$, then $\pi$ is cohomologous to $\alpha$. Besides that $\pi$ has a special form with respect to the dynamical system $\left(X, \mu, \bigcup_{i=1}^{\infty} \widehat{\Gamma}_{2}\right)$.

Q.E.D.

The following uniqueness theorem shows that any two cohomology classes of cocycles of an approximately finite full group [D] of type $\mathrm{II}_{1}$ with dense range in a given amenable group differ from each other by an automorphism in the normalizer $N[\mathscr{D}]$ of the full group. The existence of cocycles with dense ranges was established in [20], [14], [15].

Theorem 1.5. Let $G$ be an amenable l.c.s. group, $\alpha, \beta: \mathscr{D} \times X \rightarrow G$ the cocycles of a free approximately finite action of a countable group $\mathscr{D}$ on a Lebesgue space $(X, \mu)$ with invariant probability measure $\mu$. Suppose that the skew product actions $G \times{ }_{\alpha} X$ and $G \times{ }_{\beta} X$ are ergodic. Then there exist cocycles $\bar{\alpha}$ and $\bar{\beta}$ cohomologous to $\alpha$ and $\beta$ respectively as the cocycles of the full group [D] , and an automorphism $\theta \in N[\mathscr{D}]$ such that $\bar{\alpha}(\gamma, x)=\bar{\beta}\left(\theta \gamma \theta^{-1}\right.$, $\theta x)$ for all $\gamma \in[\mathscr{D}]$ at a.a. $x \in X$.

Proof. In virtue of Lemma 1.3 one may assume that $\alpha$ and $\beta$ take values in a countable dense subgroup $H \subset G$. We shall reduce simultaneously $\alpha$ and $\beta$ to a special form, and a significant part of our argument will resemble some phases of the proof of Lemma 1.4. There- 
fore we shall omit details referring the reader to the corresponding steps of the proof of the previous theorem.

Denote by $\left\{B_{i}\right\}_{i=1}^{\infty},\left\{\gamma_{i}\right\}_{i=1}^{\infty}$ and $\left\{W_{i}\right\}_{i=1}^{\infty}$ the same as in Lemma 1.4.

Fulfil for a cocycle $\alpha$ the first step of the construction described in the proof of the preceeding lemma. Thus, we construct the pairwise commuting automorphisms $\delta_{i}{ }^{a}$ on $X$ with order $2,1 \leq i \leq m_{2}$. For every $i$ fix a fundamental set $F_{i}$ of the group $\Gamma_{i}{ }^{\alpha}$, generated by $\delta_{1}{ }^{a}, \cdots, \delta_{i}{ }^{a}$, so that $F_{1} \supset$ $F_{2} \supset \cdots \supset F_{m_{2}}, \mu\left(F_{i}\right)=1 / 2^{i}$. The group $\left[\Gamma_{m_{2}}^{a}\right]$ approximates $\gamma_{1}$ in metric $d$ up to $1 / 2$, and $\Gamma_{m_{2}}^{a}$-invariant finite $\sigma$-algebra generated by $F_{m_{2}}$ approximates $B_{1}$ in measure up to $1 / 2$. Associate to the constructed above $\delta_{i}{ }^{a}$ the functions $f_{i}^{\alpha}(x)$ such that

$$
f_{i}{ }^{\alpha}(x)=\alpha\left(\delta_{i}{ }^{\alpha}, x\right) \text { for } x \in F_{i} \text {, and } f_{i}{ }^{\alpha}\left(\delta_{j}{ }^{\alpha} x\right)=f_{i}{ }^{\alpha}(x) \text { for } j \leq i .
$$

Now for every function $f_{i}{ }^{\alpha}(x)$ form the related function $p_{i}{ }^{\alpha}(x)$ just as in Lemma 1.4 constant on the sets $\gamma F_{m_{2}}, \gamma \in \Gamma_{m_{2}}^{\alpha}$, and invariant with respect to $\Gamma_{i}^{a}$, with values in a finite set $Q_{i} \subset H, e \in Q_{i}$, and such that

$$
f_{i}{ }^{\alpha}(x) \cdot p_{i}{ }^{\alpha}(x)^{-1} \in U_{i} \quad \text { for } \quad x \in A_{i}{ }^{\alpha} \text {, and } \mu\left(A_{i}{ }^{\alpha}\right)>1-1 / 2^{i}
$$

with $U_{i}$ a neighbourhood of the identity in $G$ chosen so that $h U_{i} h^{-1} \subset W_{i}$ for all $h \in Q_{1} \cdot Q_{2} \cdots Q_{i-1}$.

Turn to the cocycle $\beta$. For $i$ as above construct using the ergodicity of the skew product action $G \times{ }_{\beta} X$, the isomorphisms $\delta_{i}{ }^{\beta} \in[\mathscr{D}]$ which take $F_{i}$ onto $F_{i-1} \backslash F_{i}\left(F_{0}=X\right)$ and such that

$$
\begin{aligned}
& \beta\left(\delta_{i}^{\beta}, x\right) \in U_{i} p_{i}{ }^{\alpha}(x) \quad \text { for } \quad x \in F_{i}, \\
& \delta_{i}^{\beta}\left(\delta_{i+1}^{\alpha}\right)^{\xi_{i+1}}\left(\delta_{i+2}^{\alpha}\right)^{\xi_{i+2} \ldots\left(\delta_{m_{2}}\right)^{\xi_{m_{2}}} F_{m_{2}}} \\
& \quad=\delta_{i}{ }^{\alpha}\left(\delta_{i+1}^{\alpha}\right)^{\xi_{i+1}}\left(\delta_{i+2}^{\alpha}\right)^{\xi_{i+2}} \ldots\left(\delta_{m_{2}}^{\alpha}\right)^{\xi_{m_{2}}} F_{m_{2}}
\end{aligned}
$$

for any collection $\left\{\zeta_{j}\right\}_{j=i+1}^{m_{2}}$ of 0 's and 1's.

Thus we obtain the automorphisms $\delta_{i}^{\beta}$ on $F_{i-1}$ with order 2 which then are extended onto $\gamma F_{i-1}$ by the formulae $\gamma^{-1} \delta_{i}{ }^{\beta} \gamma$ for all elements $\gamma$ of the group $\Gamma_{i-1}^{\beta}$ generated by $\delta_{1}{ }^{\beta}, \cdots, \delta_{i-1}^{\beta}$. Set also $p_{i}{ }^{\beta}(x)=p_{i}{ }^{\alpha}(x)$ for $x \in F_{i}$ and extend $p_{i}^{\beta}(x)$ onto $X$ by $\Gamma_{i}^{\beta}$-invariance. Let $f_{i}^{\beta}(x), 1 \leq i \leq m_{2}$ be the functions associated to $\beta$ and $\delta_{1}{ }^{\beta}, \cdots, \delta_{m_{2}}^{\beta}$, i.e.

$$
f_{i}^{\beta}(x)=\beta\left(\delta_{i}{ }^{\beta}, x\right) \text { for } x \in F_{i} \text {, and } f_{i}{ }^{\beta}\left(\delta_{j}{ }^{\beta} x\right)=f_{i}^{\beta}(x) \text { for } j \leq i,
$$

then it follows from (1.10) that

$$
f_{i}^{\beta}(x) \cdot p_{i}{ }^{\beta}(x)^{-1} \in U_{i} \text { for a.a. } x \in X .
$$


Moreover, (1.11) implies the following correlation:

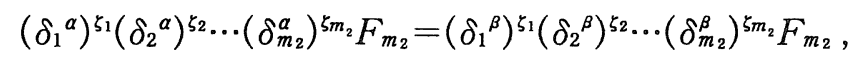

which means exactly that $p_{i}{ }^{\beta}(x)$ satisfy the conditions of Definition 1.1 with respect to the cocycle $\beta$ and the transformations $\delta_{1}{ }^{\beta}, \cdots, \delta_{m_{2}}^{\beta}$.

Further fulfil the first step of the construction described in the proof of Lemma 1.4 for the cocycle $\beta$, i.e., form the automorphisms $\delta_{i}{ }^{\beta} \in[\mathscr{D}]$ with order $2, m_{2}+1 \leq i \leq M_{2}$ which commute in pairs with each other and with $\delta_{i}{ }^{\beta}$ 's constructed before, associate to them the functions $f_{i}^{\beta}(x)$ and $p_{i}{ }^{\beta}(x)$ so that

$$
f_{i}^{\beta}(x) p_{i}{ }^{\beta}(x)^{-1} \in U_{i} \quad \text { for } \quad x \in A_{i} \text {, and } \mu\left(A_{i}{ }^{\beta}\right)>1-1 / 2^{i} .
$$

Fix also the fundamental sets $F_{m_{2}+1} \supset \cdots \supset F_{M_{2}}$ for each of the groups $\Gamma_{m_{2+1}}^{\beta}$ $\subset \cdots \subset \Gamma_{M_{2}}^{B}$ so that $F_{m_{2}+1} \subset F_{m_{2}}$.

The group $\left[\Gamma_{M_{2}}^{\beta}\right]$ approximates $\gamma_{1}$ in metric $d$ up to $1 / 2$, and the $\Gamma_{M_{2}}^{\beta}$-invariant finite $\sigma$-algebra generated by $F_{M_{2}}$ approximates $B_{1}$ in measure up to $1 / 2$.

Now for $m_{2}+1 \leq i \leq M_{2}$ and the fundamental sets $F_{i}$ constructed above form the automorphisms $\delta_{i}{ }^{\alpha} \in[\mathscr{D}]$ and the functions $f_{i}{ }^{\alpha}(x), p_{i}{ }^{\alpha}(x)$ so that

$$
\begin{aligned}
& f_{i}^{\alpha}(x)^{\alpha} p_{i}^{\alpha}(x)^{-1} \in U_{i} \text { at a.a. } x \in X, \text { and } \\
& \left(\delta_{m_{2}+1}^{\alpha}\right)^{\xi_{m_{2}}+1} \cdots\left(\delta_{M_{2}}^{\alpha}\right)^{\zeta_{M_{2}}} F_{M_{2}}=\left(\delta_{m_{2}+1}^{\beta}\right)_{\zeta_{2}+1} \cdots\left(\delta_{M_{2}}^{\beta}\right)^{\zeta_{M_{2}}} F_{M_{2}}
\end{aligned}
$$

(see the described above construction of $\delta_{i}{ }^{\beta}, p_{i}{ }^{\beta}(x), f_{i}^{\beta}(x)$ for $1 \leq i \leq m_{2}$ ).

This completes the first step of our procedure.

In a similar way construct at the $k$-th step for $M_{k}+1 \leq i \leq m_{k+1}$ the automorphisms $\delta_{i}{ }^{a}$ and the functions $p_{i}{ }^{\alpha}(x)$ which satisfy (1.9) and provide approximation of $\gamma_{k}$ and $B_{k}$ up to $1 / 2^{K}$. Then for the same $i$ form the automorphisms and the funotions $p_{i}^{\beta}(x)$ satisfying the conditions (1.12) and

$$
\left(\delta_{M_{k}+1}^{\alpha}\right)_{\zeta_{k+1}+1} \cdots\left(\delta_{m_{k+1}}^{\alpha}\right)^{\zeta m_{k+1}} F_{m_{k+1}}=\left(\delta_{M_{k}+1}^{\beta}\right)^{\zeta M_{k+1}} \cdots\left(\delta_{m_{k+1}}^{\beta}\right)^{\zeta m_{k+1}} F_{m_{k+1}} .
$$

Further construct for $m_{k+1}+1 \leq i \leq M_{k+1}$ the automorphisms $\delta_{i}{ }^{\beta}$ which provide approximation of $\gamma_{k}$ and $B_{k}$ up to $1 / 2^{k}$ and satisfy the condition (1.13). After that form the automorphisms $\delta_{i}{ }^{a}$ and the functions $p_{i}{ }^{\alpha}(x)$ which satisfy the conditions (1.14) and

$$
\begin{aligned}
& \left(\delta_{m_{k+1}+1}^{\alpha}\right)^{\zeta m_{k+1}+1} \cdots\left(\delta_{M_{k+1}}^{\alpha}\right)^{\zeta M_{k+1}} F_{M_{k+1}} \\
& \quad=\left(\delta_{m_{k+1}+1}^{\beta}\right)^{\zeta m_{k+1}} \cdots\left(\delta_{M_{k+1}}^{\beta}\right)^{\zeta m_{k+1}} F_{M_{k+1}} .
\end{aligned}
$$


The approximation of $\gamma_{i}, i \in N$, provides the coincidence of the full groups $\left[\bigcup_{i=1}^{\infty} \Gamma_{i}^{\alpha}\right],\left[\bigcup_{i=1}^{\infty} \Gamma_{i}^{\beta}\right]$ and $[\mathscr{D}]$.

Let $\bar{\alpha}$ and $\bar{\beta}$ be the cocycles corresponding respectively to $\delta_{i}{ }^{\alpha}, p_{i}{ }^{\alpha}(x)$ and $\delta_{i}{ }^{\beta}, p_{i}{ }^{\beta}(x)$. Then it follows from (1.9), (1.12), (1.13), (1.14) and Lemma 1.2 that $\bar{\alpha}$ is cohomologous to $\alpha$, and $\bar{\beta}$ is cohomologous to $\beta$.

The approximation of the Borel $\sigma$-algebra on $X$ we have made in our construction implies that almost every point $x \in X$ coincides with the intersection of all the cylinder sets corresponding to the group $\bigcup_{i=1}^{\infty} \Gamma_{i}^{\alpha}$, which contains this point:

$$
x=\bigcap_{i=1}^{\infty} \gamma^{(i)} F_{i},
$$

with $\gamma^{(i)} \in \Gamma_{i}^{\alpha}$. Evidently, this intersection is non-void iff $\gamma^{(1)} F_{1} \supset \gamma^{(2)} F_{2} \supset \cdots$, and the latter condition is equivalent to the following one:

$$
\gamma^{(i)}=\left(\delta_{i}{ }^{\alpha}\right)^{\xi_{i} \ldots}\left(\delta_{1}{ }^{\alpha}\right)^{\zeta_{1}}
$$

for some sequence $\left\{\zeta_{i}\right\}_{i=1}^{\infty} \in\{0,1\}^{N}$. Thus we have the Borel isomorphism $\theta_{\alpha}: X \rightarrow\{0,1\}^{N}$ that takes a.e. point $x \in X$ into the associated to $x$ by (1.17) sequence $\left\{\zeta_{i}\right\}_{i=1}^{\infty} \in\{0,1\}^{N}$.

Let $\theta_{\beta}: X \rightarrow\{0,1\}^{N}$ be a similar isomorphism related to the group $\bigcup_{i=1}^{\infty} \Gamma_{i}^{\beta}$. Then $\theta_{\alpha} \delta_{i}{ }^{\alpha} \theta_{\alpha}{ }^{-1}=\theta_{\beta} \delta_{i}{ }^{\beta} \theta_{\beta}{ }^{-1}=\delta_{i}$, with $\delta_{i}, i \in N$, being the automorphism of $\{0$, $1\}^{N}$ given by

$$
\left(\delta_{i}\right)_{k}= \begin{cases}s_{k}, & i \neq k \\ s_{k}+1(\bmod 2), & i=k .\end{cases}
$$

Let $\Gamma$ be the transformation group generated by $\delta_{i}$. Form the cocycles $\alpha^{\prime}$, $\beta^{\prime}: \Gamma \times\{0,1\}^{N} \rightarrow G:$

$$
\begin{aligned}
& \alpha^{\prime}(\omega, s)=\bar{\alpha}\left(\theta_{a}^{-1} \omega \theta_{\alpha}, \theta_{a}^{-1} s\right), \\
& \beta^{\prime}(\omega, s)=\bar{\beta}\left(\theta_{\beta}^{-1} \omega \theta_{\beta}, \theta_{\beta}^{-1} s\right), \omega \in[\Gamma], s \in\{0,1\}^{N} .
\end{aligned}
$$

These cocycles are determined by the functions $p_{i}{ }^{\alpha} \circ \theta_{a}{ }^{-1}$ and $p_{i}{ }^{\beta} \circ \theta_{\beta}{ }^{-1}, i \in N$, respectively (see (1.1)), and have the special form. But (1.15) and (1.16) imply $p_{i}{ }^{\alpha} \circ \theta_{\alpha}{ }^{-1}=p_{i}{ }^{\beta} \circ \theta_{\beta}{ }^{-1}$, hence $\alpha^{\prime}=\beta^{\prime}$. Thus we get a correlation

$$
\bar{\alpha}\left(\theta_{\alpha}^{-1} \omega \theta_{\alpha}, \theta_{\alpha}^{-1} s\right)=\bar{\beta}\left(\theta_{\beta}^{-1} \omega \theta_{\beta}, \theta_{\beta}^{-1} s\right) .
$$

Set $\theta_{\alpha}{ }^{-1} s=x \in X, \theta_{\alpha}{ }^{-1} \omega \theta_{\alpha}=\gamma \in[\mathscr{D}], \theta=\theta_{\beta}{ }^{-1} \theta_{\alpha}$, and obtain finally: 


$$
\bar{\alpha}(\gamma, x)=\bar{\beta}\left(\theta \gamma \theta^{-1}, \theta x\right) .
$$

Note that $\theta \in N[\mathscr{D}]$ since $\theta \delta^{\alpha}{ }^{\alpha} \theta^{-1}=\delta_{2}{ }^{\beta}, i \in N$.

Q.E.D.

Corollary 1.6. Let $G$ be an amenable l.c.s. group, $\alpha, \beta: F \times Y \rightarrow G$ the cocycles of a free approximately finite action of a countable group $F$ on a Lebesgue space $(Y, \nu)$ with invariant measure $\nu, \nu(Y)=\infty$. Suppose that the skew product actions $G \times{ }_{\alpha} Y$ and $G \times{ }_{\beta} Y$ are ergodic. Then there exist cocycles $\bar{\alpha}$ and $\bar{\beta}$ cohomologous to $\alpha$ and $\beta$ respectively as the cocycles of the full group $[F]$, and a $\nu$-preserving automorphism $\theta \in N[F]$ such that $\bar{\alpha}(\gamma, y)$ $=\bar{\beta}\left(\theta \gamma \theta^{-1}, \theta y\right)$ for all $\gamma \in[F]$ at a.a. $y \in Y$.

Proof. The $F$-action on $Y$ is orbit equivalent to the $\Gamma \times \mathbb{Z}$-action on $X \times \mathbb{Z}$, with $(X, \mu, \Gamma)$ being the dynamical system described at the beginning of this section, and $\mathbb{Z}$ acting on itself by translations. Therefore $\alpha$ and $\beta$ can be transferred to the $\Gamma \times \mathbb{Z}$-action. Change $\alpha$ and $\beta$ to the cohomologous cocycles which do not depend on the translation of $Z$, and apply Theorem 1.5 .

Q.E.D.

\section{§2. Automorphism Groups of Ergodic Equivalence Relations and Associated von Neumann Algebras}

Measure groupoids play a significant role in the modern ergodic theory. All necessary definitions as well as the detailed exposition of related techniques are contained in [10-12, 18, 19, 24, 25, 29-32].

Let $(\Omega, Q)$ be a measure groupoid and $\alpha: G \rightarrow \operatorname{Aut}(\Omega, Q)$ an action of a 1.c.s. group $G$ by strict automorphisms of the groupoid $(\Omega, Q)$ such that the map $(g, x \mapsto) \alpha(g) x$ is Borel (the latter condition will be implicit for all actions of continuous groups on groupoids or measure spaces we shall consider below). This permits one to impose the groupoid structure on $G$ $\times \Omega$. Specifically, form the projections $r, d: G \times \Omega \rightarrow\{e\} \times \Omega^{(0)}: r(g, x)=$ $(e, \alpha(g) r(x)), d(g, x)=(e, d(x))$. These maps together with the product $(g, x)(h, y)=\left(g h,\left(\alpha\left(h^{-1}\right) x\right) y\right)$ defined when $\left(\alpha\left(h^{-1}\right) x, y\right) \in \Omega^{(2)}$, provide $G \times$ $\Omega$ with a structure of an algebraic groupoid. Note that under above definitions one has $(g, x)^{-1}=\left(g^{-1}, \alpha(g) x^{-1}\right)$. All these maps are Borel relatively to the product Borel structure an $\left(G \times \Omega,\left[\mu_{G}\right] \times Q\right)$ becomes a measure groupoid ( $\mu_{G}$ is a left Haar measure of $G$ ). We shall term this construction the semidirect product and denote it by $G\left(S_{\alpha} \Omega\right.$.

A similar definition was formulated in [32] for automorphism groups of 
topological groupoids. However, in the case of measure groupoids one needs to work with an automorphism group $G$ consisting of non-strict automorphisms, i.e. isomorphisms between some inessential reductions (i. r.) of the groupoid $\Omega$. Thereby some difficulties arise in defining the semidirect product, especially when $G$ is continuous. Nevertheless it will be shown below that a slight reorganization of the original groupoid $\Omega$ makes all the automorphisms $\alpha(g)$ constituting the $G$-action on $\Omega$ to be strict. We shall stick to the case when $\Omega$ is principal and has discrete orbits.

Let $\mathscr{D}$ be a countable non-singular transformation group on a Lebesgue space $(S, \mu), \mu(S)=1$. Given any automorphism $\theta \in N[\mathscr{D}]$, then one can form two families of Borel maps $\varphi_{\gamma}, \psi_{\gamma}: S \rightarrow S, \gamma \in \mathscr{D}: \varphi_{\gamma}(s)=\theta \gamma s, \psi_{\gamma}(s)$ $=\gamma \theta s$.

Denote by $A_{\gamma \delta} \subset S$ the (Borel) set on which $\varphi_{\gamma}$ coincides with $\psi_{\delta}$. It follows from the definition of the full group normalizer that for every $\gamma \in$ $\mathscr{D}$ the set $A_{\gamma}=\bigcup_{\delta \in \mathscr{D}} A_{\gamma \delta}$ is just the entire space $S(\bmod 0)$. The same property is certainly valid for the set $A_{\theta}=\bigcap_{\gamma \in \mathscr{D}} A_{\gamma}$. Furthermore, let $V_{\theta}=\bigcap_{n \in Z}$ $A_{\theta n}$, and then $U_{\theta}(\mathscr{D})=\bigcap_{k \in Z} \theta^{k} V_{\theta}$. Thus we get a Borel set $U_{\theta}(\mathscr{D})$ which is invariant with respect to $\theta^{n}, n \in \mathbb{Z}$, and has measure 1 . It also possesses the following property: given any two points $x, y \in U_{\theta}(\mathscr{D})$, then $x$ and $y$ are in the same $\mathscr{D}$-orbit iff $\theta x, \theta y$ are in the same $\mathscr{D}$-orbit. We shall call $U_{\theta}(\mathscr{D})$ the strictness domain for the transformation $\theta \in N[\mathscr{D}]$ with respect to $\mathscr{D}$. Note that a strictness domain for $\theta$ can be also described with respect to any countable transformation group $\mathscr{D}_{1}$ such that $\left[\mathscr{D}_{1}\right]=[\mathscr{D}]$.

Denote by $R_{\mathscr{D}}$ the Borel countable equivalence relation on $S$ associated with the $\mathscr{D}$-action.

Theorem 2.1. Let $\alpha: G \rightarrow$ Aut $(S, \mu)$ be an action of a l.c.s. group $G$ on $(S, \mu)$ such that $\alpha(g) \in N[\mathscr{D}]$ for all $g \in G$. Then there exist a Borel strictly (but not only mod 0) G-invariant equivalence relation $R \subset S \times S$ and $a$ conull Borel set $B \subset S$ such that $R$ and $R_{\mathscr{D}}$ agree when restricted to $B$.

Proof. One can easily see from the above discussion that the dependence of $U_{\alpha(g)}(\mathscr{D})$ on $g$ is Borel since the map $(g, x) \rightarrow \alpha(g) x$ and the $\mathscr{D}$-action are Borel. Hence a set $A=\left\{(g, x) \in G \times S: x \in U_{\alpha(g)}(\mathscr{D})\right\}$ is Borel in $G \times S$. Moreover, this set is conull with respect to the product measure 
$\mu_{G} \times \mu$ since $\mu\left(U_{\alpha(g)}(\mathscr{D})\right)=1$ for every $g \in G$. Apply now the Fubini's theorem in order to conclude that the set $M_{x}=\left\{g \in G: x \in U_{a(g)}(\mathscr{D})\right\}$ is conull in $G$ for a.a. $x \in S$. Let $B \subset S$ be the Borel set in $S$ with $\mu$-measure 1 consisting of the points $x$ with that property.

Form the set $C=\left\{(g, x, y) \in G \times S \times S:(\alpha(g) x, \alpha(g) y) \in R_{\mathscr{D}}\right\}$. It is Borel since $C=F^{-1}\left(R_{\mathscr{D}}\right)$ for a Borel map $F: G \times X \times X \rightarrow X \times X$ given by $F(g, x, y)=(\alpha(g) x, \alpha(g) y)$. Thus we get a Borel field of sets $L(x, y)=\{g \in$ $\left.G:(\alpha(g) x, \alpha(g) y) \in R_{\mathscr{D}}\right\}$. It is straightforward to check the following properties of $L(x, y)$ :

(i) $L(x, x)=G$ for all $x \in S$;

(ii) $L(x, y)=L(y, x)$ for all $x, y \in S$;

(iii) $L(x, z) \supset L(x, y) \cap L(y, z)$ for all $x, y, z \in S$;

(iv) $L(\alpha(h) x, \alpha(h) y)=L(x, y) h^{-1}$ for all $x, y \in S, h \in G$.

Consider a subset $R=\left\{(x, y) \in S \times S: \mu_{G}(G \backslash L(x, y))=0\right\}$ in $S \times S$ together with a function $f: S \times S \rightarrow \mathbb{R}, f(x, y)=\mu_{G}(G \backslash L(x, y))$. A Borel nature of this function follows from the fact that $C$ is Borel and Theorem 1 of $[16, \S 35]$. Therefore $R$ is a Borel set.

It follows from ( $\mathrm{i}-\mathrm{iii})$ that $R$ is an equivalence relation. (iv) implies that $R$ is strictly invariant with respect to every $h \in G$, i.e., $G R=R$.

Let $x, y \in B$ be given. If $\left.(x, y) \in R_{\mathscr{D}}\right|_{B}$, then $L(x, y) \supset M_{x} \cap M_{y}$, and hence $\mu_{G}(G \backslash L(x, y))=0$. On the contrary, if $\left.(x, y) \notin R_{\mathscr{D}}\right|_{B}$, then one has $G \backslash L(x, y) \supset M_{x} \cap M_{y}$. This means exactly that $\left.R_{\mathscr{D}}\right|_{B}=\left.R\right|_{B}$.

Q.E.D.

Remark 2.2. The equivalence relation $R$ in the above theorem is a Borel set and therefore it admits the structure of an ergodic equivalence relation [24, p. 203], and hence the structure of a measure groupoid. This is provided by a measure $\nu$ on $R$ given by $\nu=\int \nu^{x} d \mu(x), x \in S$, with $\nu^{x}(E)$ $=$ card $(E \cap\{x\} \times B)$ for each Borel $E \subset S$. Thus we obtain a principal measure groupoid $R$ which is isomorphic to the groupoid $R_{\mathscr{D}}$, though the isomorphism is not strict.

The correlation $G R=R$ permits one to raise the $G$-action $\alpha$ on $(S, \mu)$ to an action by strict automorphisms $\bar{\alpha}(g)$ of the groupoid $(R,[\nu]): \bar{\alpha}(g)(x$, $y)=(\alpha(g) x, \alpha(g) y)$ for $(x, y) \in R$. This enables one to form the semidirect product $G\left(S_{\bar{\alpha}} R\right.$ which we shall also denote by $G \Im_{\bar{\alpha}} R_{\mathscr{D}}$ and thereby associate it with the original groupoid $R_{\mathscr{D}}$.

Remark 2.3. The statement of Theorem 2.1 is also valid when the 
group $\mathscr{D}$ is continuous, and every automorphism $\alpha(g)$ is inner with respect to $R_{\mathscr{D}}$, i.e., $(\alpha(g) x, x) \in R_{\mathscr{D}}$ for a.a. $x \in S$. It suffices in this case to declare $U_{\alpha(g)}(\mathscr{D})=\left\{x \in S:(\alpha(g) x, x) \in R_{\mathscr{D}}\right\}$ to be a strictness domain for $\alpha(g)$ and transfer the proof almost literally.

Let $(\Omega, Q)$ be an orbit groupoid of an ergodic type $\mathrm{II}_{\infty}$ or III action of a countable group $\Gamma$ on a Lebesgue space $(X, \mu)$ and $\left(T \times T,\left[\mu_{T} \times \mu_{T}\right]\right)$ a transitive groupoid associated with the translation of a circle $\mathbb{T}$ on itself with Haar measure $\mu_{T}$. Form the direct product $(\mathcal{Q}, C)=(\Omega \times(\boldsymbol{T} \times \mathbb{T}), Q$ $\left.\times\left[\mu_{T} \times \mu_{T}\right]\right)$. Recall that every principal ergodic groupoid with continuous orbits is isomorphic to some groupoid of the above form [10, Theorem 6.4].

Theorem 2.4. Let $A$ be an automorphism of the groupoid $(\mathcal{Q}, C)$. Then there exist an automorphism $\theta$ of $(\Omega, Q)$ and an inner automorphism $\tau$ of $(G, C)$ such that $A=(\theta \times \mathrm{id}) \tau$.

Proof. Since $(\mathscr{Q}, C)$ is principal, $A$ is completely determined by its restriction to the unit space $\mathcal{G}^{(0)}=X \times \boldsymbol{T}$. Set $A(x, t)=\left(A_{1}(x, t), A_{2}(x, t)\right)$.

Choose $t_{0} \in \boldsymbol{T}$ so that the set $X \times\left\{t_{0}\right\}$ is contained $\bmod 0$ in the i.r. of $\mathcal{G}$ on which $A$ is a strict isomorphism. Form a Borel map $\varphi: X \rightarrow X, \varphi(x)=$ $A_{1}\left(x, t_{0}\right)$. Since $A$ is an automorphism, one may assume after discarding a Borel null set that every point in $X$ has at most countable inverse image with respect to $\varphi$. This implies that $\varphi(X)$ is a Borel subset of $X$ with positive measure. Note that the partition of $X$ into the inverse images of $\varphi$ is measurable. Hence there is a Borel set $S \subset X$ with positive measure such that $\varphi$ is one-one when restricted to $S$.

Note that $A_{1}(x, t)$ and $\varphi(x)$ are in the same $\Gamma$-orbit when $(x, t)$ is in some i.r., and hence the maps $A$ and $(x, t) \rightarrow(\varphi(x), t)$ take almost every pair $(x, t)$ into $\Gamma \times \mathbb{T}$-equivalent pairs. A is an isomorphism, hence $\varphi$ should take complete sets into complete ones, and non-complete into non-complete ones (recall that a measurable set in the unit space of a groupoid is said to be complete iff its saturation is conull). Note that the class of complete sets in $X$ is just the class of sets of positive measure due to the countability of $\Gamma$. Thus $\varphi$ is non-singular on $S$.

We shall assume below that the $\Gamma$-action preserves the measure $\mu$, and $\mu(X)=\infty$. The case of type III $\Gamma$-action can be considered in a similar way.

Let $f(x)=d \mu^{\circ} \varphi / d \mu(x)$ be the Radon-Nikodym derivative of the mea- 
sure $\mu^{\circ} \varphi$ with respect to $\mu$. One can readily deduce from the $\Gamma$-invariance of $\mu$ that $f(x)$ is invariant with respect to the full group $[\Gamma]$ reduced to $S$. By the ergodicity of $\Gamma f(x)$ is constant a.e., that is, $\mu^{\circ} \varphi=c \mu$ for some $c>$ 0 .

Replacing if necessary $S$ by its subset of finite measure and normalizing $\mu$ in a suitable way, we can get $\mu(S)=1, \mu(\varphi(S))=c$. Partition $X$ twice into countable families of disjoint sets $X=\bigcup_{i=0}^{\infty} S_{i}=\bigcup_{i=0}^{\infty} S_{i}{ }^{\prime}$ so that $S_{0}=$ $S, S_{0}{ }^{\prime}=\varphi(S), \mu\left(S_{i}\right)=1, \mu\left(S_{i}{ }^{\prime}\right)=c$. Choose the transformations $\gamma_{i}, \gamma_{i}{ }^{\prime} \in[\Gamma]$, $i=0,1,2, \cdots$ so that $\gamma_{i}\left(S_{0}\right)=S_{i}, \gamma_{i}{ }^{\prime}\left(S_{0}{ }^{\prime}\right)=S_{i}{ }^{\prime}$. Finally, set $\theta: X \rightarrow X, \theta x=\gamma_{i}{ }^{\prime}$ $\circ \varphi \circ \gamma_{i}^{-1}(x)$ for $x \in S_{i}$. Clearly $\theta \in N[\Gamma]$ and hence $\theta$ is an automorphism of $(\Omega, Q)$. It follows from the above constructions that $\theta(x)$ and $\varphi(x)$ are $\Gamma$-equivalent at a.a. $x \in X$. Thus $\theta \times$ id is pointwise $\Gamma \times \mathbb{T}$-equivalent to $\varphi$ $\times \mathrm{id}$, and hence to $A$. This implies $A \cdot(\theta \times \mathrm{id})^{-1}$ is an inner automorphism of $(G, C)$.

Q.E.D.

The paper by P. Hahn [19] presents a construction which associates to each measure groupoid $(\mathcal{H}, B)$ a Banach $*$-algebra $\operatorname{II}(\mathscr{H})$ together with its regular representation in $L^{2}(\mathscr{H})$ by convolution operators $L_{f}, f \in \operatorname{II}(\mathscr{H})$. This permits, in particular, to put in correspondence to each automorphism of the measure groupoid $\mathcal{H}$ an automorphism of the von Neumann algebra $L(\mathrm{II}(\mathscr{H}))^{\prime \prime}$, and thereby to impose the notion of module for groupoid automorphisms. We shall describe briefly the corresponding construction for ergodic type II groupoid $(\mathcal{G}, C)$ mentioned above in this section.

To begin with, consider the groupoid $(\Omega, Q)$ with discrete orbits (the discrete reduction of $(\mathcal{Q}, C)$ ). We shall assume below for simplicity's sake that $(\Omega, Q)$ is generated by a free action of a countable group $\Gamma$, so $(\Omega, Q)$ $=\left(\Gamma \times X,\left[\mu_{\Gamma} \times \mu\right]\right)$ with $\mu_{\Gamma}$ a counting measure on $\Gamma$. One has:

$$
\begin{aligned}
& \mathrm{II}(\Gamma \times X)=\left\{f \in L^{1}\left(\Gamma \times X, \mu_{\Gamma} \times \mu\right):\|f\|_{\mathrm{II}}<\infty\right\}, \\
& \|f\|_{\mathrm{II}}=\sup \left\{\sum_{\gamma \in \Gamma} \int|f(\gamma, x) j(x) k(\gamma x)| d \mu(x): \int|j|^{2} d \mu=\int|k|^{2} d \mu=1\right\} .
\end{aligned}
$$

The convolution of functions and involution are defined in the following way:

$$
\begin{aligned}
& (f * g)(\gamma, x)=\sum_{\gamma^{\prime} \in \Gamma} f\left(\gamma \gamma^{\prime-1}, \gamma^{\prime} x\right) \cdot g\left(\gamma^{\prime}, x\right), \\
& f^{*}(\gamma, x)=\overline{f\left(\gamma^{-1}, \gamma x\right)}, \quad f, g \in \operatorname{II}(\Gamma \times X) .
\end{aligned}
$$

Form type $\mathrm{II}_{\infty}$ factor $M=L(\mathrm{II}(\Gamma \times X))^{\prime \prime}$ generated by the convolution 
operators $L_{f}$ :

$$
L_{f} \xi=f * \xi, \quad f \in \operatorname{II}(\Gamma \times X), \quad \xi \in L^{2}\left(\Gamma \times X, \mu_{\Gamma} \times \mu\right) .
$$

Then every element of $M$ is an operator of convolution with some measurable function [12, propositions 2.6, 2.10].

Given any function $a(x) \in L^{\infty}(X)$, then one can form a function

$$
\tilde{a}(\gamma, x)=\left\{\begin{array}{cc}
a(x), & \gamma=e \\
0, & \gamma \neq e .
\end{array}\right.
$$

The convolution operator $L_{\tilde{a}}$ is just the multiplication operator by $a(x)$ :

$$
\left(L_{a} \xi\right)(\gamma, x)=a(\gamma x) \cdot \xi(\gamma, x) \text { for } \xi \in L^{2}(\Gamma \times X) .
$$

Let $\mathcal{A}$ be the subalgebra in $M$ consisting of all the multiplication operators, then $\mathcal{A}$ is maximum Abelian and isomorphic to $L^{\infty}(X)$. Moreover, $\mathcal{A}$ is regular in the sense that its normalizer $N(\mathcal{A})=\{U \in M: U$ is unitary, $\left.U \mathscr{A} U^{*}=\mathcal{A}\right\}$ generates $M$ [12, proposition 2.9].

Every automorphism of the groupoid $\Gamma \times X$ is determined by some $\theta \in$ $N[\Gamma]$ and acts in a following way:

$$
\theta(\gamma, x)=\left(\gamma^{\theta}(x), \theta x\right)
$$

with $\gamma^{\theta}(x) \in \Gamma$ be such that $\gamma^{\theta}(x) \theta x=\theta \gamma x . \quad \theta$ can be raised to an automorphism $\bar{\theta}$ of the Banach algebra $\operatorname{II}(\Gamma \times X)$ :

$$
(\bar{\theta} f)(\gamma, x)=f\left(\gamma^{\theta^{-1}}(x), \theta^{-1} x\right) .
$$

It is easy to see that $\bar{\theta}$ can be extended to $M$.

The following two lemmas concerning the connection between automorphisms of principal measure groupoids with countable orbits and automorphisms of the associated von Neumann algebras are due to $\mathrm{J}$. Feldman and C. C. Moore [12].

Lemma 2.5. There is a one-to-one correspondence between the inner automorphisms of the groupoid $\Gamma \times X$ (or just the elements of the full group $[\Gamma])$ and the classes of unitary operators $U \in N(\mathcal{A})$ which differ from each other by some unitary $a \in \mathcal{A}$.

Lemma 2.6. For every outer automorphism of $\Gamma \times X$ there is a naturally associated class of coincident on $\mathcal{A}$ outer automorphisms $\phi$ of $M$ such that $\phi(\mathcal{A})=\mathcal{A}$. Conversely, every class of outer automorphisms of $M$ with above properties determines some outer automorphism of $\Gamma \times X$. 
Turn to the groupoid $(\mathscr{L}, C)$, whose orbits are continuous. The associated von Neumann algebra $L(\mathrm{II}(\mathcal{Q}))^{\prime \prime}$ splits into the tensor product

$$
L(\mathrm{II}(\Gamma \times X))^{\prime \prime} \otimes L(\mathrm{II}(\mathbb{T} \times \mathbb{T}))^{\prime \prime} \cong M \otimes \mathbb{1}_{L^{2}(T)} \otimes B\left(L^{2}(\mathbb{T})\right) .
$$

It contains a maximum Abelian regular subalgebra $A_{d} \otimes A_{c}$ with $A_{d}=\mathcal{A}$, $A_{c} \cong L^{\infty}(T)$ [10, propositions 8.1, 8.2].

Theorem 2.7. There is a one-to-one correspondence between the inner automorphisms of $(\mathcal{G}, C)$ and the classes of operators from $N\left(A_{d} \otimes A_{c}\right) \subset$ $M \otimes B\left(L^{2}(T)\right)$, which differ by unitary elements of $A_{d} \otimes A_{c}$.

Proof. Let $\theta$ be an inner automorphism of $(\mathcal{Q}, C)$. The restriction of $\theta$ to the unit space $X \times \mathbb{T}$ is defined by some Borel maps $\varphi: X \times \mathbb{T} \rightarrow \Gamma$ and $\psi: X \times \mathbb{T} \rightarrow \mathbb{T}:$

$$
\theta(x, t)=(\varphi(x, t) x, \phi(x, t)) .
$$

Let $X \times \mathbb{T}=\bigcup_{\gamma \in \Gamma} Q_{r}$ be the partition of the unit space into the inverse images of $\varphi, Q_{\gamma}=\varphi^{-1}(\gamma)$. This partition induces a family of transformations $\gamma^{-1} \theta: Q_{\gamma} \rightarrow \gamma^{-1} \theta Q_{\gamma}, \gamma \in \Gamma$, submitted to the $\mathbb{T}$-action on $X \times \mathbb{T}$. These transformations generate the partial isometries $a_{r} \in A_{d} \otimes B\left(L^{2}(\mathbb{T})\right)$.

Consider the family of unitary operators $\lambda_{\gamma} \in M \otimes B\left(L^{2}(T)\right), \gamma \in \Gamma$, given by $\left(\lambda_{\gamma} \xi\right)\left(\left(\gamma^{\prime}, x\right),(s, t)\right)=\xi\left(\left(\gamma^{-1} \gamma^{\prime}, x\right),(s, t)\right)$, with $\left(\gamma^{\prime}, x\right) \in \Gamma \times X,(s, t)$ $\in \mathbb{T} \times \mathbb{T}, \xi \in L^{2}(\mathcal{Q})$.

The strongly convergent row $U_{\theta}=\sum_{\gamma \in \Gamma} \lambda_{\gamma} a_{\gamma}$ determines some unitary operator $U_{\theta} \in N\left(A_{d} \otimes A_{c}\right)$. We shall denote by $\bar{\theta}$ the associated inner automorphism Ad $U_{\theta}$ of $M \otimes B\left(L^{2}(T)\right)$.

Let now $U$ be a unitary operator from $N\left(A_{d} \otimes A_{c}\right), \tau$ the trace on $M \otimes B\left(L^{2}(\mathbb{T})\right)$ and $E$ the conditional expectation onto the subalgebra $A_{d} \otimes B\left(L^{2}(\mathbb{T})\right)$. Then $U$ admits the decomposition $U=\sum_{\gamma \in \Gamma} \lambda_{\gamma} a_{\gamma}$ with $a_{\gamma}=$ $E\left(\lambda_{\gamma^{-1}} U\right) \in A_{d} \otimes B\left(L^{2}(T)\right)$, which converges to an operator in $L^{2}\left(M \otimes B\left(L^{2}(\boldsymbol{T})\right), \tau\right)$. It follows from the orthogonality properties of the family $\left\{\lambda_{\gamma}: \gamma \in \Gamma\right\}$ with respect to $\tau$ and the condition $U \in N\left(A_{d} \otimes A_{c}\right)$ that $a_{r}$ in the decomposition of $U$ are the partial isometries. Moreover, the systems of domain and range projections of the partial isometries $\lambda_{\gamma} a_{\gamma}$ are disjoint and contained in $A_{d} \otimes A_{c}$. By means of multiplication by the unitary elements of $A_{d} \otimes A_{c}$ one can make $a_{r}$ to be generated by the $\mathbb{T}^{\prime}$ action on $X \times \mathbb{T}$. Thus we obtain a row with the structure as above for the operator $U a, a \in A_{d} \otimes A_{c}$ being a unitary element. Let $\theta$ be the 
automorphism of the Lebesgue space $X \times T$ which is the point realization of the action of $\operatorname{Ad}(U a)$ on $A_{d} \otimes A_{c}$ [23], then $\operatorname{Ad}(U a)=\bar{\theta}$.

Q.E.D.

Theorem 2.8. There is a one-to-one correspondence between the outer automorphisms of $(\mathcal{Q}, C)$ and the classes of outer automorphisms $\phi$ of $M \otimes B\left(L^{2}(T)\right)$ coincident on $A_{d} \otimes A_{c}$ and such that $\phi\left(A_{d} \otimes A_{c}\right)=A_{d} \otimes A_{c}$.

Proof. Let $A$ be an outer automorphism of $\mathcal{G}$. By Theorem 2.4 it admits a representation $A=(\theta \times \mathrm{id}) \cdot \omega$ with $\theta$ being an outer automorphism of $\Gamma \times X, \omega$ an inner automorphism of $\mathcal{G}$. Associate with $A$ the automorphism $\bar{A}=(\bar{\theta} \times \mathrm{id}) \cdot \bar{\omega}$ of $M \otimes B\left(L^{2}(\boldsymbol{T})\right)$ where $\bar{\theta}$ is the automorphism of $M$ given by (2.2) and $\bar{\omega}$ the inner automorphism of $M \otimes B\left(L^{2}(T)\right)$, described in the proof of Theorem 2.7.

Conversely, let $\phi$ be an outer automorphism of $M \otimes B\left(L^{2}(T)\right)$ such that $\phi\left(A_{d} \otimes A_{c}\right)=A_{d} \otimes A_{c} . \quad$ This clearly implies that $\phi\left(N\left(A_{d} \otimes A_{c}\right)\right)=$ $N\left(A_{d} \otimes A_{c}\right)$ and hence by Theorem 2.7 the point realization $A$ of $\phi$ on $\mathcal{G}^{(0)}$ normalizes the group of inner automorphisms. We need only to deduce from this fact that $A$ is an automorphism of $(\mathcal{Q}, C)$.

Recall that $(\mathscr{Q}, C)$ is an orbit groupoid for an ergodic action of a l.c.s. group $G=\Gamma \times \boldsymbol{T}$ on a Lebesgue space $\left(\mathcal{G}^{(0)}, \tilde{\mu}\right)=\left(X \times \boldsymbol{T}, \mu \times \mu_{T}\right)$. We shall denote by $\beta(g), g \in G$, the automorphisms which constitute this action, and $R_{\beta}$ the corresponding Borel equivalence relation on $\mathcal{G}^{(0)}$.

Form an action $\alpha: G \rightarrow \operatorname{Aut}\left(\mathcal{G}^{(0)}, \widetilde{\mu}\right)$ by the automorphisms $\alpha(g)=$ $A^{-1} \beta(g) A$, and let $R_{\alpha}$ be the corresponding Borel equivalence relation on $\mathcal{G}^{(0)}$. It follows from the above discussion that every transformation $\alpha(g)$ is inner with respect to $R_{\beta}$. We apply Theorem 2.1 and Remark 2.3 to deduce the existence of a Borel equivalence relation $R$ on $\mathcal{G}^{\left({ }^{(0)}\right.}$ and a conull Borel set $B \subset \mathcal{G}^{(0)}$ such that $\alpha(g) R=R$ strictly for all $g \in G$, and $\left.R\right|_{B}=\left.R_{\beta}\right|_{B}$. This implies, in particular, that every transformation $\alpha(g)$ is inner also with respect to $R$, i.e. for every $g \in G$ a Borel set $E_{g}=\left\{x \in \mathcal{G}^{(0)}:(\alpha(g) x, x)\right.$ $\in R\}$ is conull in $\mathcal{G}^{(0)}$. Thus the Borel set $E=\left\{(g, x) \in G \times \mathcal{G}^{(0)}:(\alpha(g) x, x)\right.$ $\in R\}$ is $\mu_{G} \times \tilde{\mu}$-conull in $G \times \mathcal{G}^{(0)}$ and hence by Fubini's Theorem the set $E_{x}$ $=\{g \in G:(\alpha(g) x, x) \in R\}$ is conull in $G$ for $x$ in some conull Borel $B^{\prime} \subset \mathcal{Q}^{(0)}$. It follows from the strict $\alpha$-invariance of $R$ that $E_{x}$ is a conull subgroup in $G$, and hence $E_{x}=G$ for $x \in B^{\prime}$. This means exactly that $\left.\left.R_{\alpha}\right|_{B^{\prime}} \subset R\right|_{B^{\prime}}$. Form a Borel set $B_{1}=B \cap B^{\prime}$, then $\left.R\right|_{B_{1}}=\left.R_{\beta}\right|_{B_{1}}$, and hence $\left.\left.R_{\alpha}\right|_{B_{1}} \subset R_{\beta}\right|_{B_{1}}$. One can readily exchange the actions $\alpha$ and $\beta$ with each other in the above reasoning and get a conull Borel set $B_{2} \subset \mathcal{G}^{(0)}$ such that $\left.\left.R_{\beta}\right|_{B_{2}} \subset R_{\alpha}\right|_{B_{2}}$. Thus 
we have proved that the equivalence relations $R_{\alpha}$ and $R_{\beta}$ coincide when restricted to the conull Borel set $B_{1} \cap B_{2}$. But $R_{\alpha}=A\left(R_{\beta}\right)$, and hence $R_{\beta}$ is $A$-invariant $\bmod 0$, i.e., $A$ is an automorphism of $(\mathscr{L}, C)$. It is easy to see that $\phi$ and $\bar{A}$ agree on $A_{d} \otimes A_{c}$.

Definition 2.9. We call the number $\bmod A=\tau \circ \bar{A} / \tau$ a module of an automorphism $A$ of type II groupoid $(\mathcal{Q}, C)$ described in this section, where $\bar{A}$ is the automorphism of $M \otimes B\left(L^{2}(\mathbb{T})\right)$ associated to $A, \tau$ the trace on $M \otimes B\left(L^{2}(T)\right)$. Now let $(\mathscr{H}, P)$ be an arbitrary type II groupoid with continuous orbits, and $\varphi: \mathcal{H} \rightarrow \mathcal{Q}$ an isomorphism. For an automorphism $B$ of $(\mathcal{H}, P)$ set up $\bmod B=\bmod \left(\varphi B \varphi^{-1}\right)$.

Evidently, module is a homomorphism Aut $\left.(\mathscr{H}, P) \rightarrow \mathbb{R}_{+}{ }^{*}\right)$ which does not depend on the choice of $\varphi$.

Remark 2.10. The module of an automorphism $A$ of $(\mathcal{G}, C)$ coincide with the module of the associated to $A$ by Theorem 2.4 automorphism $\theta \in$ $N[\Gamma], \bmod \theta=\mu \circ \theta / \mu$.

Let $\left(\Omega_{1}, Q_{1}\right)$ be an orbit groupoid of an ergodic type $\mathrm{II}_{1}$ action of a countable group $\mathscr{D}$ on a Lebesgue space $(S, \nu)$. Form the direct product $\left(\mathcal{Q}_{1}, C_{1}\right)=\left(\Omega_{1} \times(T \times T), Q_{1} \times\left[\mu_{T} \times \mu_{T}\right]\right)$. It is certainly isomorphic to some groupoid $(y, C)$ as described before in this section, whose discrete reduction $(\Omega, Q)$ is type $\mathrm{II}_{\infty}$. The following can be easily deduced from Theorem 2.4 .

Corollary 2.11. Let $A$ be an automorphism of the groupoid $\left(\mathcal{Q}_{1}, C_{1}\right)$, and $\bmod A=1$. Then there exist an automorphism $\theta$ of the groupoid $\left(\Omega_{1}\right.$, $\left.Q_{1}\right)$ and an inner automorphism $\omega$ of $\left(\mathcal{Q}_{1}, C_{1}\right)$ such that $A=(\theta \times \mathrm{id}) \omega$.

Recall that the automorphism $\theta \in N[\mathscr{D}]$ for an arbitrary full group [D] is said to be inner if $\theta \in[\mathscr{D}]$ and outer otherwise. In a similar way, the action $\alpha: G \rightarrow$ Aut $(S, \nu)$ of a l.c.s. group $G$ such that $\alpha(g) \in N[\mathscr{D}]$ for all $g$ $\in G$, is called outer if every transformation $\alpha(g)$ is outer. A sharpening of this notion is given by

Definition 2.12. The action $\alpha$ is said to be strictly outer if there exists a conull Borel set $B \subset S$ such that for all $s \in B \alpha(g)_{s} \in B$ and $\alpha(g)_{s}=\gamma$ for some $\gamma \in \mathscr{D}$ implies $\gamma=e_{\mathscr{D}}, g=e_{G}$.

Clearly every strictly outer action is outer and free. Moreover, the semidirect product $G\left(\Im_{\bar{\alpha}} R_{\mathscr{D}}\right.$ formed in Remark 2.2 is a principal groupoid 
iff the action $\alpha$ is strictly outer.

It is easy to see that every outer action of a countable group $G$ is also strictly outer, but this is not the case for a continuous group $G$.

Example 2.13. We shall show how to imbed an arbitrary l.c.s. group $G$ into the normalizer $N[\mathscr{D}]$ of a type $\mathrm{II}_{1}$ full group [D] so that the $G$-action is strictly outer. Consider the case of noncompact $G$.

Let $(Y, \nu)$ be a free properly ergodic (i.e. non-transitive) Lebesgue $G$ space with invariant probability measure $\nu$. Form the space $(X, \mu)=(Y$, $\nu)^{\mathscr{D}}$ which admits a $\mu$-preserving $G$-action by the automorphisms $\alpha(g)$ :

$$
(\alpha(g) x)_{r}=g x_{\gamma}, \quad \gamma \in \mathscr{D} .
$$

Define also a free ergodic $\mu$-preserving $\mathscr{D}$-action on $(X, \mu)$ :

$$
(\gamma x)_{\lambda}=x_{\delta \gamma}, \quad \gamma, \delta \in \mathscr{D} .
$$

This $\mathscr{D}$-action clearly commutes with $\alpha(g), g \in G$, and so $\alpha(g) \in N[\mathscr{D}]$. We claim the action $\alpha$ is strictly outer.

Suppose $\alpha(g) x=\gamma x$ for some $x \in X, g \in G, \gamma \in \mathscr{D}$. Since the actions of $G$ and $\mathscr{D}$ are free, one may assume that $g \neq e_{G}, \gamma \neq e_{\mathscr{D}}$. Then $x$ satisfies the condition $x_{\delta \gamma}=g x_{\delta}$ for all $\delta \in \mathscr{D}$, and hence is contained in the Borel set $B_{\gamma}=\left\{x \in X: x_{\gamma} \in G x_{e}\right\}$ where $G x_{e}$ is the $G$-orbit of $x_{e} \in Y$. Since the $G$ action on $Y$ is properly ergodic, every $G$-orbit in $Y$ has $\nu$-measure 0 , hence $\mu\left(B_{\gamma}\right)=0$. Set up $B=\bigcup_{\gamma \in \mathscr{D}} B_{\gamma}$, then $\mu(B)=0$. This means that the action $\alpha$ is strictly outer.

A strictly outer action of an arbitrary compact group $G$ can be constructed in a similar way.

\section{§ 3. Outer Conjugacy for Actions of Unimodular Amenable Groups}

We shall denote throughout this section by $\Gamma$ a free countable amenable ergodic transformation group of a Lebesgue space $(S, \mu), \mu(S)=1$, with $\mu$ being $\Gamma$-invariant. $\quad \alpha, \beta: G \rightarrow$ Aut $(S, \mu)$ will mean the actions of a continuous unimodular amenable 1.c.s. group $G$ on $(S, \mu)$ by the automorphisms $\alpha(g), \beta(g) \in N[\Gamma]$. Recall that such actions are called outer conjugate if $\alpha(g)=\varphi \beta(g) \varphi^{-1} t(g)$ for some $\varphi \in N[\Gamma]$ and $t(g) \in[\Gamma]$ for all $g \in G$.

Consider the groupoid $(\mathcal{Q},[\nu])$ generated by the $\Gamma$-action on $S: \mathcal{Q}=\Gamma$ $\times S, \nu=\mu_{\Gamma} \times \mu$. Then by Theorem 2.1 and Remark 2.2 the actions $\alpha$ and $\beta$ 
generate actions of $G$ by the strict automorphisms $\bar{\alpha}(g)$ and $\bar{\beta}(g)$ of some groupoid $R$ isomorphic to $\mathcal{G}$, which we shall identify with $\mathcal{G}$.

Let $(\mathscr{P},[\eta])$ be the transitive groupoid generated by the translation of $G, \mathscr{Q}=G \times G, \eta=\mu_{G} \times \mu_{G}$. Consider the direct product $(\mathcal{Q} \times \mathscr{Q},[\rho]), \rho=\nu$ $\times \eta$. The automorphisms $\bar{\alpha}(g), \bar{\beta}(g)$ are naturally extended up to automorphisms $\widetilde{\alpha}(g)=\bar{\alpha}(g) \times$ id and $\tilde{\beta}(g)=\bar{\beta}(g) \times$ id of $\mathcal{Q} \times \mathscr{Q}$.

Definition 3.1. We shall say that the actions $\alpha$ and $\beta$ are stably outer conjugate if there exist an automorphism $A$ and a Borel family of inner automorphisms $l(g)$ of the groupoid $\mathcal{Q} \times \mathscr{Q}$ such that for every $g \in G$ the automorphisms $\tilde{\beta}(g)$ and $A \widetilde{\alpha}(g) A^{-1} l(g)$ agree on an i.r. of $\mathcal{Q} \times \mathscr{Q}$.

Lemma 3.2. Every two strictly outer actions $\alpha$ and $\beta$ are stably outer conjugate.

Proof. Let $\tau: G \rightarrow G \times G$ be a Borel measure preserving isomorphism of the Lebesgue spaces. Define the map $\zeta: \mathscr{D} \rightarrow \mathscr{Q} \times \mathscr{P}$ by

$$
\zeta(g, h)=\left(\tau_{1}(g h) \cdot \tau_{1}(h)^{-1}, \tau_{1}(h)\right) \times\left(\tau_{2}(g h) \cdot \tau_{2}(h)^{-1}, \tau_{2}(h)\right),
$$

where $\tau(g)=\left(\tau_{1}(g), \tau_{2}(g)\right)$. It is easy to see that $\zeta$ is a strict isomorphism of groupoids, which can be extended up to a strict isomorphism $\tilde{\zeta}: \mathcal{G} \times \mathscr{D} \rightarrow \mathscr{G}$ $\times \mathscr{D} \times \mathscr{Q}, \tilde{\zeta}=\mathrm{id} \times \zeta$. This isomorphism transfers the $G$-action onto the groupoid $(\mathcal{Q} \times \mathscr{Q} \times \mathscr{Q},[\nu \times \eta \times \eta])$. Specifically, we have for each $g \in G$ the automorphisms $\widehat{\alpha}(g)=\tilde{\zeta} \widetilde{\alpha}(g) \tilde{\zeta}^{-1}$ and $\widehat{\beta}(g)=\widetilde{\zeta} \widetilde{\beta}(g) \tilde{\zeta}^{-1}$ given by

$$
\begin{aligned}
& \widehat{\alpha}(g)(x, s, t)=(\bar{\alpha}(g) x, s, t), \\
& \widehat{\beta}(g)(x, s, t)=(\bar{\beta}(g) x, s, t)
\end{aligned}
$$

for $(x, s, t) \in \mathcal{Q} \times \mathscr{Q} \times \mathscr{Q}$.

Form the semidirect products $\left(G \Im_{\tilde{a}}(\mathcal{Q} \times \mathscr{Q}),\left[\mu_{G} \times \nu \times \eta\right]\right)$ and $\left(G\left(S_{\bar{\beta}}(\mathcal{Q}\right.\right.$ $\left.\times \mathscr{Q}),\left[\mu_{G} \times \nu \times \eta\right]\right)$ together with their homomorphisms $\pi_{\alpha}$ and $\pi_{\beta}$ respectively into $G: \pi_{\alpha}(g, \tilde{x})=g$ for $(g, \tilde{x}) \in G \Im_{\tilde{\alpha}}(\mathscr{Q} \times \mathscr{L}) ; \pi_{\beta}$ is defined in a similar way. This permits one to consider the skew products

$$
\begin{aligned}
& \left(G \times{ }_{\pi_{a}} G\left(_{\tilde{\alpha}}(\mathcal{G} \times \mathscr{P})\right),\left[\mu_{G} \times \mu_{G} \times \nu \times \eta\right]\right) \text { and } \\
& \left(G \otimes_{\pi_{\beta}}\left(G\left(\Im_{\bar{\beta}}(\mathscr{Q} \times \mathcal{P})\right),\left[\mu_{G} \times \mu_{G} \times \nu \times \eta\right]\right)[10] .\right.
\end{aligned}
$$

Each of them possesses the $G$-action $\omega_{\alpha}$ and $\omega_{\beta}$ respectively:

$$
\omega_{a}(p)(h, g, x, t)=\left(h p^{-1}, g, x, t\right)
$$

for $(h, g, x, t) \in G \times_{\pi_{\alpha}}\left(G\left(S_{\tilde{\alpha}}(\mathscr{Q} \times \mathscr{D})\right) ; \omega_{\beta}(p)\right.$ is defined in a similar way. 
Let the action of $G$ by inner automorphisms $\hat{l}(g)$ on $\mathcal{G} \times \mathscr{P} \times \mathscr{Q}$ be given by $\hat{l}(g)(x, t,(k, h))=\left(x, t,\left(k, h g^{-1}\right)\right)$ for $x \in \mathcal{G}, t \in \mathscr{Q},(k, h) \in \mathscr{Q}$.

Define the maps $\phi_{a}: G \times{ }_{\pi_{\alpha}}\left(G\left(S_{\tilde{\alpha}}(\mathcal{Q} \times \mathscr{L})\right) \rightarrow \mathscr{Q} \times \mathscr{Q} \times \mathscr{Q}\right.$,

$$
\begin{aligned}
& \phi_{\alpha}(h, g, x, t)=\left(\bar{\alpha}\left(h^{-1}\right) x, t,(g, h)\right), \text { and } \\
& \phi_{\beta}: G \times_{\pi_{\beta}}\left(G S_{\bar{\beta}}(\mathcal{Q} \times \mathscr{P})\right) \rightarrow \mathcal{G} \times \mathscr{P} \times \mathscr{Q}, \\
& \phi_{\beta}(h, g, x, t)=\left(\bar{\beta}\left(h^{-1}\right) x, t,(g, h)\right), \text { for } x \in \mathcal{Q}, t \in \mathscr{P}, \\
& (g, h) \in \mathscr{Q} .
\end{aligned}
$$

One can readily check that $\phi_{a}$ and $\phi_{\beta}$ are the groupoid isomorphisms and the following is true:

$$
\begin{aligned}
& \widehat{\alpha}(g) \hat{l}(g)=\phi_{\alpha} \omega_{\alpha}(g) \phi_{\alpha}{ }^{-1} ; \\
& \widehat{\beta}(g) \hat{l}(g)=\phi_{\beta} \omega_{\beta}(g) \phi_{\beta}{ }^{-1} .
\end{aligned}
$$

The groupoids $G\left(S_{\tilde{\alpha}}(\mathscr{Q} \times \mathscr{P})\right.$ and $G S_{\tilde{\beta}}(\mathscr{Q} \times \mathscr{Q})$ may be written in the form $\left(G\left(S_{\bar{\alpha}} \mathcal{Q}\right) \times \mathscr{P}\right.$ and $\left(G \mathbb{S}_{\bar{\beta}} \mathcal{L}\right) \times \mathscr{P}$ respectively, and they should be approximately finite due to the amenability of $G$ (see the Appendix). Moreover, since the actions $\alpha$ and $\beta$ are strictly outer, the groupoids above should be principal. Hence by [10, Theorem 6.4] there exist the isomorphisms

$$
\begin{aligned}
& F_{\alpha}: G\left(\mathbb{S}_{\bar{\alpha}}(\mathcal{Q} \times \mathscr{P}) \rightarrow(\mathbb{Z} \times \boldsymbol{T}) \times\left(S^{\prime} \times T\right)\right. \\
& F_{\beta}: G\left(S_{\bar{\beta}}(\mathcal{L} \times \mathscr{P}) \rightarrow(\mathbb{Z} \times T) \times\left(S^{\prime} \times T\right)\right.
\end{aligned}
$$

Since $G$ is unimodular, it follows that the modular homomorphisms [18] of the groupoids $G\left(S_{\bar{\alpha}}(\mathscr{Q} \times \mathscr{Q})\right.$ and $G\left(S_{\bar{\beta}}(\mathscr{Q} \times \mathscr{Q})\right.$ are trivial, that is both groupoids are of type II. Hence we may identify $\mathbb{Z}$-actions on $S^{\prime}$, written in (3.3) and (3.4), and choose them to be of type $\mathrm{II}_{\infty}$.

This permits one to transfer $\pi_{\alpha}$ and $\pi_{\beta}$ onto the groupoid $(Z \times \mathbb{T}) \times\left(S^{\prime}\right.$ $\times T)$. Set up $\bar{\pi}_{\alpha}=\pi_{\alpha} \circ F_{\alpha}{ }^{-1}, \bar{\pi}_{\beta}=\pi_{\beta} \circ F_{\beta}{ }^{-1}$ and form the corresponding skew products $G \times \bar{\pi}^{a}\left((Z \times T) \times\left(S^{\prime} \times T\right)\right), \quad G \times \bar{\pi}^{\beta}\left((Z \times T) \times\left(S^{\prime} \times T\right)\right) . \quad F_{a}, F_{\beta}$ are naturally extended up to the isomorphisms between the skew products:

$$
\begin{aligned}
& \bar{F}_{\alpha}: G \times_{\pi_{a}}\left(G S_{\bar{a}}(\mathcal{Q} \times \mathscr{Q})\right) \rightarrow G \times_{\bar{\pi}_{a}}\left((Z \times T) \times\left(S^{\prime} \times T\right)\right) \\
& \bar{F}_{\beta}: G \times_{\pi_{\beta}}\left(G S_{\bar{\beta}}(\mathcal{G} \times \mathscr{Q})\right) \rightarrow G \times{ }_{\bar{\pi}_{\beta}}\left((\boldsymbol{Z} \times \boldsymbol{T}) \times\left(S^{\prime} \times \mathbb{T}\right)\right) .
\end{aligned}
$$

These induce the actions of $G$ by the automorphisms

$$
\bar{\omega}_{\alpha}(g)=\bar{F}_{\alpha} \omega_{\alpha}(g) \bar{F}_{\alpha}^{-1} \text { and } \bar{\omega}_{\beta}(g)=\bar{F}_{\beta} \omega_{\beta}(g) \bar{F}_{\beta}^{-1}
$$


on $G \times \times_{\bar{\pi}_{a}}\left((\mathbb{Z} \times \mathbb{T}) \times\left(S^{\prime} \times \mathbb{T}\right)\right)$ and $G \times_{\bar{\pi}_{\beta}}\left((\mathbb{Z} \times \mathbb{T}) \times\left(S^{\prime} \times \mathbb{T}\right)\right)$, respectively.

It is easy to see that the cocycles $\bar{\pi}_{\alpha}$ and $\bar{\pi}_{\beta}$ have the dense ranges in $G$. Using a simple argument, we can replace them by cohomologous cocycles independent of the translation of $\mathbb{T}$. Thus we may apply Corollary 1.6 in order to deduce the existence of a Borel function $f: S^{\prime} \times \mathbb{T} \rightarrow G$ and an automorphism $\theta \in N[\mathbb{Z}]$ such that (see (2.1))

$$
\left.f(n \cdot s, r t)^{-1} \bar{\pi}_{\alpha}((n, r),(s, t))\right) f(s, t)=\bar{\pi}_{\beta}\left(\left(n^{\theta}(s), r\right),(\theta s, t)\right)
$$

on some i.r. of $(\mathbb{Z} \times \mathbb{T}) \times\left(S^{\prime} \times \mathbb{T}\right)$. Define the map

$$
\phi: G \times \bar{\pi}_{a}\left((\mathbb{Z} \times \mathbb{T}) \times\left(S^{\prime} \times \mathbb{T}\right)\right) \rightarrow G \times \bar{\pi}_{\beta}\left((\mathbb{Z} \times \mathbb{T}) \times\left(S^{\prime} \times \mathbb{T}\right)\right)
$$

by $\phi(g,(n, r),(s, t))=\left(f(s, t)^{-1} g,\left(n^{\theta}(s), r\right),(\theta s, t)\right)$. Then $\phi$ is a groupoid isomorphism, and

$$
\bar{\omega}_{\beta}(g)=\phi \bar{\omega}_{\alpha}(g) \phi^{-1}
$$

for all $g \in G$ on some i.r.

Combine now (3.1), (3.2), (3.5), (3.6) to get the correlation $\tilde{\beta}(g) \tilde{l}(g)=$ $A \widetilde{\alpha}(g) \tilde{l}(g) A^{-1}$, with $\tilde{l}(g)=\tilde{\zeta}^{-1} \hat{l}(g) \tilde{\zeta}$, and $A=\tilde{\zeta}^{-1} \phi_{\beta} \bar{F}_{\beta}{ }^{-1} \phi \bar{F}_{\alpha} \phi_{\alpha}{ }^{-1} \tilde{\zeta}$ being an automorphism of $\mathcal{Q} \times \mathscr{P}$. Equivalently,

$$
\tilde{\beta}(g)=A \widetilde{\alpha}(g) A^{-1} l(g)
$$

for some Borel family $l(g)$ of inner automorphisms of $\mathcal{Q} \times \mathscr{P}$. (3.7) holds for every fixed $g \in G$ on some i.r. of $\mathcal{G} \times \mathscr{Q}$.

Q.E.D.

Theorem 3.3. Every two strictly outer actions $\alpha$ and $\beta$ are outer conjugate.

Proof. Return to the groupoid $G \times \times_{\bar{\pi}_{a}}\left((\mathbb{Z} \times \mathbb{T}) \times\left(S^{\prime} \times \mathbb{T}^{\prime}\right)\right)$ we have considered when proving Lemma 3.2. Replace the cocycle $\bar{\pi}_{\alpha}$ by a cohomologous one $\pi$, independent of the translation of $\mathbb{T}$, and respectively, pass to the isomorphic skew product. The latter may be written in the form $\left(G \times{ }_{\pi}\left(\mathbb{Z} \times S^{\prime}\right)\right) \times(\mathbb{T} \times \mathbb{T})$.

For an arbitrary transformation $\theta_{1} \in N[\mathbb{Z}]$ form the cocycle $\pi^{\theta_{1}}(n, s)=$ $\pi\left(n^{\theta_{1}}(s), \theta_{1} s\right)$ (see (2.1)). Evidently, $\pi^{\theta_{1}}$ has the dense range in $G$. Hence by Corollary 1.6 there exist a Borel function $f_{\theta_{1}}: S^{\prime} \rightarrow G$ and $\theta_{0} \in N[\mathbb{Z}]$ such that $\bmod \theta_{0}=1$ and $f_{\theta_{1}}(n \cdot s)^{-1} \pi^{\theta_{1}}(n, s) f_{\theta_{1}}(s)=\pi\left(n^{\theta_{0}}(s), \theta_{0} s\right)$.

Define the automorphism $\phi_{\theta_{1}}$ of the skew product

$$
\begin{aligned}
& G \times{ }_{\pi}\left(\mathbb{Z} \times S^{\prime}\right): \\
& \phi_{\theta_{1}}(g,(n, s))=\left(f_{\theta_{1}}\left(\theta_{1}^{-1} s\right)^{-1} g,\left(n^{\theta_{0} \theta_{1}-1}(s), \theta_{0} \theta_{1}^{-1} s\right) .\right.
\end{aligned}
$$


Evidently, $\bmod \phi_{\theta_{1}}=\left(\bmod \theta_{1}\right)^{-1}$. This induces naturally the automorphism $\bar{\phi}_{\theta_{1}}$ of the groupoid $G \times \times_{\bar{\pi}_{a}}\left((\mathbb{Z} \times T) \times\left(S^{\prime} \times T\right)\right)$ commuting with the $G$-action $\bar{\omega}_{a}$ and such that $\bmod \bar{\phi}_{\theta_{1}}=\left(\bmod \theta_{1}\right)^{-1}$. Thus we can replace correlation (3.6) by the equivalent one:

$$
\bar{\omega}_{\beta}(g)=\phi \bar{\phi}_{\theta_{1}} \bar{\omega}_{\alpha}(g) \bar{\phi}_{\theta_{1}}^{-1} \phi^{-1}
$$

and then $A$ in (3.7) by the automorphism

$$
\begin{aligned}
& B=\tilde{\zeta}^{-1} \phi_{\beta} \bar{F}_{\beta}^{-1} \phi \bar{\phi}_{\theta_{1}} \bar{F}_{\alpha} \phi_{\alpha}{ }^{-1} \tilde{\zeta}, \text { so that } \\
& \tilde{\beta}(g)=B \tilde{\alpha}(g) B^{-1} l^{\prime}(g)
\end{aligned}
$$

for some Borel family $l^{\prime}(g)$ of inner automorphisms of $\mathcal{Q} \times \mathscr{Q}$. Choosing $\theta_{1}$ $\in N[\boldsymbol{Z}]$ in a proper way, we can get $\bmod B=1$.

By Corollary 2.11 there are $\varphi \in N[\Gamma]$ and an inner automorphism $\tau$ of $\mathcal{Q} \times \mathscr{P}$ such that $B=(\varphi \times$ id $) \tau$. Rewrite (3.9) in the form

$$
\bar{\beta}(g) \times \mathrm{id}=(\varphi \times \mathrm{id})(\bar{\alpha}(g) \times \mathrm{id})\left(\varphi^{-1} \times \mathrm{id}\right) l^{\prime \prime}(g) .
$$

This implies that the family $l^{\prime \prime}(g)$ of inner automorphisms should have the form $l^{\prime \prime}(g)=p(g) \times$ id with $p(g) \in[\Gamma]$ for each $g \in G$. Hence $\beta(g)=$ $\varphi \alpha(g) \varphi^{-1} p(g)$.

Q.E.D.

Remark 3.4. A similar argument proves Theorem 3.3 in the case when the approximately finite full group $[\Gamma]$ is of type $\mathrm{II}_{\infty}$, and $\bmod \alpha(g)=\bmod$ $\beta(g)=1$ for all $g \in G$. Moreover, the intertwining automorphism $\varphi \in N[\Gamma]$ in this case has module 1 .

\section{$\S 4$. Outer Conjugacy of Compact Groups}

Let $\Gamma, S, \mu, \alpha, \beta$ be the same as in $\S 3$. We replace only an arbitrary continuous amenable unimodular l.c.s. group $G$ by a compact second countable group $K$, and prove a sharpening of Theorem 3.3 for strictly outer actions $\alpha$ and $\beta$ of $K$. It turns out that two such actions are simply conjugate by means of some transformation from $N[\Gamma]$.

Theorem 4.1. For every two strictly outer actions $\alpha$ and $\beta$ of $K$ there exists an automorphism $\varphi \in N[\Gamma]$ such that $\beta(g) s=\varphi \alpha(g) \varphi^{-1} s$ for all $g \in K$ at a.a. $s \in S$.

Proof. It follows from the compactness of $K$ that the partitions of $S$ into $\alpha$-or $\beta$-orbits are measurable. Moreover, since $\Gamma$ is ergodic and $\alpha, \beta$ 
are strictly outer, every $\alpha$ - or $\beta$-orbit is a null Borel set. This implies that the orbit spaces $S / \alpha(K)$ and $S / \beta(K)$ are standard and uncountable, hence isomorphic. Set $S / \alpha(K)=S / \beta(K)=X$, and denote by $\varphi_{\alpha}, \varphi_{\beta}: X \rightarrow S$ the corresponding Borel sections.

Define the Borel isomorphisms $\psi_{a}, \psi_{\beta}: K \times X \rightarrow S: \psi_{a}(g, x)=\alpha\left(g^{-1}\right) \varphi_{a}(x)$, $\psi_{\beta}(g, x)=\beta\left(g^{-1}\right) \varphi_{\beta}(x)$ for $(g, x) \in K \times X$. Let also the $K$-action on $K \times X$ be given by $\omega(g)(h, x)=\left(h g^{-1}, x\right)$. It is easy to check the following correlations:

$$
\begin{aligned}
& \omega(g)=\psi_{\alpha}{ }^{-1} \alpha(g) \psi_{\alpha}, \\
& \omega(g)=\psi_{\beta}{ }^{-1} \beta(g) \psi_{\beta} .
\end{aligned}
$$

Let $R_{\Gamma} \subset S \times S$ be the equivalence relation on $S$ generated by the $\Gamma$-action. Then Theorem 2.1 implies the existence of a Borel equivalence relation $R$ which agree with $R_{r}$ when restricted to some conull Borel set $B$ $\subset S$ and such that

$$
\alpha(K) R=R .
$$

Consider the set of pairs $R_{\alpha} \subset X \times X:\left(x_{1}, x_{2}\right) \in R_{\alpha}$ if there exists $\pi_{\alpha}\left(x_{1}\right.$, $\left.x_{2}\right) \in K$ such that

$$
\left(\alpha\left(\pi_{\alpha}\left(x_{1}, x_{2}\right)^{-1}\right) \varphi_{\alpha}\left(x_{1}\right), \varphi_{\alpha}\left(x_{2}\right)\right) \in R .
$$

Lemma 4.2. $R_{a}$ is a Borel equivalence relation with an inessential discrete reduction, and $\pi_{\alpha}$ is a Borel cocycle of $R_{\alpha}$.

Proof. Since the action $\alpha$ is strictly outer, one may assume, possibly after discarding from $S$ a $\alpha(K)$-invariant Borel null set, that each pair $\left(x_{1}\right.$, $\left.x_{2}\right) \in R_{\alpha}$ determines uniquely $\pi_{\alpha}\left(x_{1}, x_{2}\right) \in K$. It is straightforward to deduce from the condition $\alpha(K) R=R$ that $R_{\alpha}$ is an equivalence relation and $\pi_{\alpha}$ is a cocycle. We shall show in more details that $R_{\alpha}$ and $\pi_{\alpha}$ are Borel.

Form the Borel function $f: K \times X \times X \rightarrow S \times S$ :

$$
f\left(k, x_{1}, x_{2}\right)=\left(\alpha(k) \varphi_{\alpha}\left(x_{1}\right), \varphi_{\alpha}\left(x_{2}\right)\right),
$$

together with projections $p_{X \times X}: K \times X \times X \rightarrow X \times X, p_{k}: K \times X \times X \rightarrow K$. Since the projection $p_{X \times X}$ is injective when restricted to $f^{-1}(R)$, the equivalence relation $R_{\alpha}=p_{X \times X}\left(f^{-1}(R)\right)$ is Borel. Furthermore, for every Borel set $C \subset K$ the inverse image $\pi_{\alpha}{ }^{-1}(C)=p_{X \times X}\left(p_{k}{ }^{-1}(C) \cap f^{-1}(R)\right)$ is Borel, hence $\pi_{\alpha}$ is a Borel map. 
The equivalence relation $\mathcal{E}$ generated by $R$ and the action $\alpha$ of $K$ on $S$, admits the structure of a measure groupoid (see Remark 2.2). Hence by [31, Theorem 5.6] it has a discrete reduction, i.e. a Borel $\mathcal{E}$-complete set $Y_{1}$ $\subset S$ whose intersection with each equivalence class is at most countable. Replacing if necessary $Y$ by its $\mathcal{E}$-complete Borel subset, one may assume that $Y$ meets every $\alpha$-orbit at most once. It follows from the correlation $\alpha(K) R=R$ that the $\mathcal{E}$-saturation of $Y_{1}$ coincides with the $R$-saturation of $\alpha(K) Y_{1}$. Since the latter saturation is $S(\bmod 0)$, and $R$ agree with $R_{\Gamma}$ on a conull set $B \subset S$, the set $\alpha(K) Y_{1}$ should have the positive measure in $S$.

Now apply the above argument to the reduction of $\mathcal{E}$ by $S \backslash \alpha(K) Y_{1}$ and thereby obtain the set $Y_{2}$, then form in a similar way $Y_{3}$ etc. Let $Y=$ $\bigcup_{i=1}^{\infty} Y_{i}$, then the reduction of $\mathcal{E}$ to $Y$ has at most countable equivalence classes. After discarding a Borel $\alpha$-invariant null set the projection of $S$ onto the quotient space $X$ becomes one-to-one when restricted to $Y$. This projection provides an isomorphism between $\left.\mathcal{E}\right|_{Y}$ and $R_{a}$, hence $R_{\alpha}$ has an inessential discrete reduction.

Q.E.D.

Completion of the proof of Theorem 4.1. $\quad R_{\alpha}$ induces an equivalence relation $R_{a}{ }^{K}$ on $K \times X:\left(g_{1}, x_{1}\right) \sim\left(g_{2}, x_{2}\right)$ if $\left(x_{1}, x_{2}\right) \in R_{a}$ and $g_{1}=\pi_{a}\left(x_{1}, x_{2}\right) g_{2}$. The map $\psi_{\alpha} \times \psi_{\alpha}$ is an isomorphism between $R_{\alpha}{ }^{K}$ and $R$, hence $R_{\alpha}{ }^{K}$ is Borel. In virtue of Lemma 4.2 and [11, Theorem 1] we may assume that $R_{\alpha}$ is generated by an action of a countable group $\mathscr{D}_{\alpha}$ on $X$, and so $R_{a}{ }^{K}$ is generated by a skew product action $K \times{ }_{\pi_{\alpha}} X$ with finite invariant measure $\left(\psi_{a}^{-1}\right)_{*} \mu$.

Let $\nu_{a}$ be the projection of the measure $\left(\psi_{a}^{-1}\right)_{*} \mu$ onto $X$, then $\mathscr{D}_{\alpha}$-action is ergodic and $\nu_{\alpha}$-preserving. Since, due to $\psi_{a}$, the transformations $\omega(g), g \in K$, should preserve the measure $\left({\psi_{a}}^{-1}\right)_{*} \mu$, the latter admits the decomposition $\left(\psi_{a}{ }^{-1}\right)_{*} \mu=\mu_{k} \times \nu_{a}$, with $\mu_{k}$ being the Haar measure of $K$.

$\psi_{a}$ provides also the orbit equivalence between the $\Gamma$-action on $S$ and the skew product action $K \times \pi_{\alpha} X$, hence the latter is ergodic and approximately finite (equivalently, amenable [5]) with respect to the measure $\left(\psi_{\alpha}^{-1}\right)_{*}$. Therefore the $\mathscr{D}_{\alpha}$-action on $X$ is also approximately finite [36, Proposition 2.6].

Form in a similar way an equivalence relation $R_{\beta}$ on $X$, a cocycle $\pi_{\beta}$ with dense range in $K$, and a measure $\nu_{\beta}$. Since $R_{\beta}$ is also a countable approximately finite type $\mathrm{II}_{1}$ ergodic equivalence relation, one may assume that up to a Borel automorphism of $X, R_{\alpha}=R_{\beta}=R_{X}, \nu_{\alpha}=\nu_{\beta}=\nu$. Suppose 
that $R_{X}$ is generated by an ergodic $\nu$-preserving automorphism $A$.

Since the skew product actions $K \times_{\pi_{\alpha}} X$ and $K \times_{\pi_{\beta}} X$ are ergodic, we may apply Theorem 1.5 in order to deduce the existence of $\theta \in N[A]$ and a Borel function $f: X \rightarrow K$ such that $f(\delta x)^{-1} \pi_{\alpha}(\delta, x) f(x)=\pi_{\beta}\left(\theta^{-1} \delta \theta, \theta^{-1} x\right)$ for all $\delta \in[A]$ at a.a. $x \in X$.

Define the map $\phi: K \times X \rightarrow K \times X$ by $\phi(g, x)=\left(f(x)^{-1} g, \theta^{-1} x\right)$. It is Borel, $\mu_{k} \times \nu$-preserving and provides an orbit equivalence between the skew product actions $K \times_{\pi_{\alpha}} X$ and $K \times_{\pi_{\beta}} X$. Clearly, $\phi$ commutes with $\omega(g), g \in K$. Using this fact and (4.1), (4.2), we get $\beta(g)=$ $\psi_{\beta} \phi \psi_{\alpha}{ }^{-1} \alpha(g) \psi_{\alpha} \phi^{-1}{\psi_{\beta}}^{-1}$ with $\psi_{\beta} \phi \psi_{\alpha}{ }^{-1} \in N[\Gamma]$.

Q.E.D.

Remark 4.3. Suppose that the $\Gamma$-action in Theorem 4.1 preserves infinite (but $\sigma$-finite) measure $\mu$. Then it follows from the compactness of $K$ that $\bmod \alpha(g)=\bmod \beta(g)=1$ for all $g \in K$. Thus the proof of Theorem 4.1 may be repeated almost literally in the case of transformation group $\Gamma$ of type $\mathrm{II}_{\infty}$.

\section{Appendix}

The following theorem generalizes a well known proposition by $\mathrm{A}$. Connes and W. Krieger [6].

Theorem A.1. Let $(S, \mu)$ be a Lebesgue space with a probability measure $\mu, T$ an ergodic non-singular transformation of $(S, \mu)$. Given also an action of an amenable l.c.s. group $G \subset N[T]$ on $(S, \mu)$. Then the equivalence relation $R$ on $S$, generated by $G$ and $T$, is approximately finite.

In virtue of Theorem 2.1 and Remark $2.2 R$ admits the structure of a measure groupoid.

Before proving Theorem A.1 note, that by [5], it suffices to check the amenability of $R$. The definition of amenability for equivalence relations given below is just the rephrasing of the Definition 1.4 from [36] (see also [25, p. 205]).

Let $E$ be a separable Banach space, and for each $s \in S$ one has a non-void weakly-*-compact convex set $K_{s}$ in $E^{*}$. Then $s \mapsto K_{s}$ will be called a Borel field of weakly-*-compact convex sets if the set $\{(s, f): f \in$ $\left.K_{s}\right\}$ is Borel in $S \times E^{*}$ with $E^{*}$ being supplied with a Borel structure generated by the weak-*-topology.

A measured equivalence relation $R$ on $S$ is called amenable if for every 
separable Banach space $E$, for every homomorphism $\varphi$ from $R$ into the group ISO $(E)$ of isometric automorphisms of $E$, given the strong operator topology, and for every Borel field $s \mapsto K_{s}$ of weakly-*-compact convex sets in $E^{*}$ which is invariant under $\varphi$ in the sense that

$$
\varphi^{*}\left(s_{1}, s_{2}\right) K_{s_{2}}=K_{s_{1}} \text { for a.a. }\left(s_{1}, s_{2}\right) \in R
$$

with $\varphi^{*}\left(s_{1}, s_{2}\right)=\varphi\left(s_{1}, s_{2}\right)^{*-1}$, there is a Borel function $e: S \rightarrow E^{*}$ such that $e(s)$ $\in K_{s}$ and $\varphi^{*}\left(s_{1}, s_{2}\right) e\left(s_{2}\right)=e\left(s_{1}\right)$.

Proof of Theorem A.1. Let $E, \varphi$, and $s \mapsto K_{s}$ be the same as above. Consider $L^{1}(S, E)=\left\{f: S \rightarrow E, f\right.$ weakly measurable, and $\left.\int\|f(s)\| d \mu(s)<\infty\right\}$, where the functions which agree a.e. are identified. This is a separable Banach space with the norm $\|f\|=\int\|f(s)\| d \mu(s)$ [9]. Define the (strongly continuous) representation $\pi$ of $G$ in the group of isometric automorphisms of $L^{\infty}(S, E)$ :

$$
(\pi(g) h)(s)=\varphi\left(s, g^{-1} s\right) h\left(g^{-1} s\right) \frac{d \mu \circ g^{-1}}{d \mu}(s),
$$

for $h \in L^{1}(S, E)$. The adjoint representation $\pi^{*}$ operates in the dual space $L^{\infty}\left(S, E^{*}\right)=\left\{f: S \rightarrow E^{*}, f\right.$ weakly-*-measurable, and

$$
\text { ess sup }\|f(s)\|<\infty\}
$$

[9, Theorem 8.18.2] in the following way:

$$
\left(\pi^{*}(g) f\right)(s)=\varphi^{*}\left(s, g^{-1} s\right) f\left(g^{-1} s\right)
$$

for $f \in L^{\infty}\left(S, E^{*}\right)$, that is, $\pi^{*}(g)=\pi\left(g^{-1}\right)^{*}$.

Consider the set

$$
K=\left\{f: S \rightarrow E^{*}, f \text { weakly-* } \text {-measurable, and } f(s) \in K_{s}\right\} .
$$

Lemma A.2. $K \subset L^{\infty}\left(S, E^{*}\right)$, and is contained in some ball.

Proof. It suffices to show that a.a. $K_{s}$ lie in some ball in $E^{*}$. For this, consider the function $r: S \rightarrow \boldsymbol{R}, r(s)=\sup \left\{\|f\|: f \in K_{s}\right\}$ and check its measurability. Define also the Borel maps $\psi: S \times E^{*} \rightarrow \boldsymbol{R}, \psi(s, f)=\|f\|$ (this map is Borel due to the weak-*-compactness of the unit ball), and $p: S \times E^{*} \rightarrow S$ (the projection), $p(s, f)=s$. Then for any $c>0$ the set $r^{-1}((c,+\infty))=$ $p\left(\psi^{-1}((c,+\infty)) \cap\left\{(s, f): f \in K_{s}\right\}\right)$ is measurable since $s \mapsto K_{s}$ is Borel. That is, $r$ is a measurable map.

Furthermore, it follows from (A.1) that $r(s)$ is invariant with respect 
to the ergodic transformation $T$, hence $r(s)$ is constant a.e.

Q.E.D.

Completion of the proof of Theorem A.1. It follows from Lemma A. 2 and [36, Proposition 2.2] that $K$ is weakly-*-compact convex set in $L^{\infty}(S$, $\left.E^{*}\right)$. By (A.1) it is invariant with respect to $\pi^{*}(g), g \in G$.

Define an isometric operator $A$ in $L^{1}(S, E)$ :

$$
(A h)(s)=\varphi(s, T s) h(T s) \frac{d \mu \circ T}{d \mu}(s)
$$

for $h \in L^{1}(S, E)$. The adjoint operator $A^{*}$ is also isometric. It is easy to see that the subspace fix $A^{*}=\left\{f \in L^{\infty}\left(S, E^{*}\right): A^{*} f=f\right\}$ is closed in the weak- * topology. A simple argument based on the correlation $G \subset N[T]$ shows that fix $A^{*}$ is invariant under $\pi^{*}(g), g \in G$.

Resuming the above observations, we see that $K_{T}=K \cap$ fix $A^{*}$ is a weakly-*-compact convex $\pi^{*}$-invariant set in $L^{\infty}\left(S, E^{*}\right)$.

$K_{T}$ is non-void since for $A^{*}$ (hence for the associated affine $Z$-action) there exists a fixed point in the weakly-*-compact convex set $K$. Since $G$ is amenable, it possesses a fixed point in $K_{T}$. This is just the desired invariant section for $\varphi^{*}$ and the field $s \mapsto K_{s}$, and so the amenability of $R$ is proved.

Q.E.D.

\section{References}

[1] Bezuglyi, S. I. and Golodets, V. Ya., Groups of measure space transformations and invariants of outer conjugation for automorphisms from normalizers of type III full groups, J. Funct. Anal., 60 (1985), 341-369.

[2] - Outer conjugacy for actions of countable amenable groups on a measure space (in Russian), Izvestiya Acad. Sci. USSR, ser. Math., 50 (1986), 643-660.

[3] - Type III transformations of measure space and outer conjugation of countable amenable groups of automorphisms, Inst. Low Temperat. Phys. \& Engin. UkrSSR Acad. Sci., Kharkov, 1985, preprint No 28-85.

[4] Connes, A., Outer conjugacy classes of automorphisms of factors, Ann. Sci. Ec. Norm. Sup., 8 (1975), 383-420.

[5] Connes, A., Feldman, J. and Weiss, B., An amenable equivalence relation is generated by a single transformation, Ergod. Theory and Dyn. Syst., 1 (1981), 431-450.

[6] Connes, A. and Krieger, W., Measure space automorphisms, the normalizers of their full groups, and approximate finiteness, J. Funct. Anal., 24 (1977), 336-352.

[ 7 ] Cornfeld, I.P., Fomin, S. V. and Sinai, Ya. G., Ergodic Theory, New York e.a., Springer, 1982.

[ 8 ] Dye, H.A., On groups of measure preserving transformations I, Amer. J. Math., 1 (1959), 119-159.

[9] Edwards, R. E., Functional Analysis, Holt, Rinehart \& Winston, New York, 1965. 
[10] Feldman, J., Hahn, P. and Moore, C.C., Orbit structure and countable sections for actions of continuous groups, Adv. in Math., 28 (1978), 186-230.

[11] Feldman, J. and Moore, C.C., Ergodic equivalence relations, cohomology, and von Neumann algebras I, Trans, Amer. Math. Soc., 234 (1977), 289-324.

[12] - Ergodic equivalence relations, cohomology, and von Neumann algebras II, Trans. Amer. Math. Soc., 234 (1977), 325-359.

[13] Golodets, V. Ya., Description of representations of anticommutation relations (in Russian), Uspekhi Mat. Nauk, 24 (1969), 3-64.

[14] Golodets, V. Ya. and Sinelschchikov, S. D., Existence and uniqueness of cocycles of an ergodic automorphism with dense ranges in amenable groups, Inst. Low Temperat. Phys. \& Engin. UkrSSR Acad. Sci., Kharkov, 1983, preprint No 19-83.

[15] - Locally compact groups appearing as ranges of cocycles of ergodic $Z$-actions, Ergod. Theory and Dyn. Syst., 5 (1985), 47-57.

[16] Halmos, P. R., Measure Theory, Van Nostrand, Princeton N. J., 1962.

[17] Hamachi, T., Oka, Yu. and Osikawa, M., Flows associated with ergodic non-singular transformation groups, Publ. RIMS, Kyoto Univ., 11 (1975), 31-50.

[18] Hahn, P., Haar measure for measure groupoids, Trans. Amer. Math. Soc., 242 (1978), 1-34.

[19] —- The regular representations of measure groupoids, Trans. Amer. Math. Soc., 242 (1978), 35-72.

[20] Herman, M., Constructions des diffeomorphismes ergodiques, preprint.

[21] Jones, V. F. R. and Takesaki, M., Actions of compact Abelian groups on semifinite injective factors, Acta Math., 153 (1984), 213-258.

[22] Krieger, W., On ergodic flows and isomorphism of factors, Math. Ann., 223 (1976), 19-70.

[23] Mackey, G. W., Point realizations of transformation groups, Illinois J. Math., 6 (1962), 327-335.

[24] - Ergodic theory and virtual groups, Math. Ann., 166 (1966), 187-207.

[25] Moore, C.C., Ergodic theory and von Neumann algebras, Proc. Symp. Pure Math., 38 (1982), part 2, 179-226.

[26] Ocneanu, A., Actions of discrete amenable groups on von Neumann algebras, Lect. Notes Math., 1138 (1985), 1-114.

[27] Ornstein, D.S., Ergodic Theory, Randomness and Dynamical Systems, Yale Univ. Press, New Haven and London, 1974.

[28] Ornstein, D.S. and Weiss, B., Ergodic theory of amenable group actions I: the Rohlin lemma, Bull. Amer. Math. Soc., 2 (1980), 161-164.

[29] Ramsay, A., Virtual groups and group actions, Adv. in Math., 6 (1971), 253-322.

[30] - Subobjects of virtual groups, Pacif. J. Math., 87 (1980), 389-454.

[31] - Topologies on measured groupoids, J. Funct. Anal., 47 (1982), 314-343.

[32] Renault, J., A groupoid approach to $C^{*}$-algebras, Lect. Notes Math., 793 (1980), 1-160.

[33] Rubshtein, B.A. and Fedorov, A. L., On subgroups of a full ergodic approximately finite group of a measure preserving Lebesgue space automorphisms (in Russian), Funkts. Analiz $i$ yego Prilozh., 20 (1986), 82-83.

[34] Stepin, A.M., On the cohomology of Lebesgue space automorphism groups (in Russian), Funkts, Analiz i yego Prilozh., 5 (1971), 91-92.

[35] Sutherland, C.E. and Takesaki, M., Actions of discrete amenable groups on von Neumann algebras, Publ. RIMS, Kyoto Univ., 21 (1985), 1087-1120.

[36] Zimmer, R.J., Amenable ergodic group actions and an application to Poisson boundaries of random walks, J. Funct. Anal., 27 (1978), 350-372.

[37] Golodets, V. Ya. and Sinelshchikov, S.D., Outer conjugacy for actions of continuous 
amenable groups, Inst. Low Temper. Phys. \& Engin. UkrSSR Acad. Sci., Kharkov, 1985, preprint No 21-85.

[38] Sinelshchikov, S. D., Conjugacy of compact automorphism groups of ergodic equivalence relations, (in Russian), Dokl. UkrSSR Acad. Sci., 1986, 21-23. 
